\title{
Removal of Dust Microelectric Signal Based on Empirical Mode Decomposition and Multifractal Detrended Fluctuation Analysis
}

\author{
Jiming Li $(\mathbb{D}$, Yongji Sun $(\mathbb{D}$, and Xuezhen Cheng $(\mathbb{D}$ \\ College of Electrical Engineering and Automation, Shandong University of Science and Technology, 579 Qianwangang Road, \\ Huangdao District, Qingdao 266590, Shandong, China
}

Correspondence should be addressed to Xuezhen Cheng; zhenxc6411@163.com

Received 25 June 2021; Accepted 27 July 2021; Published 9 August 2021

Academic Editor: Yu-Ting Bai

Copyright (c) 2021 Jiming Li et al. This is an open access article distributed under the Creative Commons Attribution License, which permits unrestricted use, distribution, and reproduction in any medium, provided the original work is properly cited.

Microcharge induction has recently been applied as a dust detection method. However, in complex environments, the detection device can be seriously polluted by noise. To improve the quality of the measured signal, the characteristics of both the signal and the noise should be analyzed so as to determine an effective noise removal method. Traditional removal methods mostly deal with specific noise signals, and it is difficult to consider the correlation of measured signals between adjacent time periods. To overcome this shortcoming, we describe a method in which wavelet decomposition is applied to the measured signal to obtain sub-band components in different frequency ranges. A time-lapse Pearson method is then used to analyze the correlation of the sub-band components and the noise signal. This allows the sub-band component of the measurement signal that has the strongest correlation with the noise to be determined. Based on multifractal detrended fluctuation analysis combined with empirical mode decomposition, the similarity between the signal sub-band components and the noise sub-band components is analyzed and three indices are employed to determine the multifractal characteristics of the sub-band components. The consistency between noise components and signal components is obtained and the main signal components are verified. Finally, the sub-band components are used to reconstruct the signal, giving the noise-free measured (microcharge induction) signal. The filtered signal presents smoother, multifractal features.

\section{Introduction}

One of the notable properties of dust is its electrification during movement. There has been considerable research on the chargeability of dust and how to measure the electrostatic signal [1-6], and it has been widely used in the detection of gas flow velocity in the field of pneumatic conveying. Yan et al. [7-9] conducted extensive research on electrostatic induction for measuring gas-solid two-phase flows and proposed various types of measurement electrodes and measurement methods. In recent years, soft measurement and machine learning [10] have been applied to measure the parameters of gas-solid two-phase flows by the induced charge method. Zheng et al. [11] studied the electrification of sand dust and the spatial variation of the particle concentration along the flow direction by assuming a certain charge-to-mass ratio. Xu et al. $[12,13]$ proposed a new type of electrostatic sensor system for measuring the pulverized coal speed and relative mass flow, which determines the speed of pulverized coal from the autocorrelation of the output signal of the electrostatic sensor array. The relative concentration and mass flow rate are then obtained from the root mean square value output by two ring electrodes. The present authors [6] have conducted research on the theory of mine dust belt motors, charging modes, charging measurement models, and measuring devices and have identified nonlinear characteristics in the dust electrostatic signal that reflect the multiscale information contained in the signal. Because the measurement environment of the detection device is complex and changeable, the measured dust electrostatic signal always contains noise. The noise that exists when the detection device is working is also called measurement noise (in the remainder of this paper, the electrostatic signal of dust is referred to as the 
measurement signal). This noise seriously affects the measurement accuracy and must be removed.

The processing of electrostatic signals obtained by electrostatic sensors is mostly achieved by adding anti-aliasing or low-pass filters to the hardware circuit. There are relatively few signal processing methods for electrostatic signals. Wang et al. [14] compared the frequency spectra of the measured signals from external-surface electrostatic sensors and embedded electrostatic sensors and used a harmonic wavelet transform to decompose the measured signal. The frequency spectrum of inner flush-mounted electrostatic sensors was found to contain a critical frequency for decoupling the induced charge signal from the transferred charge signal, thus improving the cross-correlation speed of the signal. Kamel and Yan [15] proposed a hybrid removal method that combines the cut-off frequency method for removing the noise component outside the signal bandwidth with a median filter for smoothing the signal, thus minimizing the effect of noise in the signal. Wang [16] proposed a radial vibration measurement method based on electrostatic sensing and Hilbert-Huang transform (HHT) signal processing, which provides a simple and economical method for rotating-shaft radial vibration measurements. Zhang [17] used the HHT to study the characteristics of different electrodes' electrostatic signals and extracted the features that characterize the changes in solid concentration. Wang et al. [18] proposed an adaptive decomposition method based on the discrete wavelet transform (DWT), which effectively reduces the average relative standard deviation of the solid velocity. The abovementioned studies can be combined with modern signal processing methods to achieve electrostatic signal processing, but the basic characteristics and frequency range of noise signals are preset, and so adaptive noise recognition and processing cannot be performed. To remove noise, it is necessary to separate the signal from the noise, or to identify the obvious differences in their respective characteristics. The multilayer wavelet decomposition transform is an effective signal-noise separation method in both the time domain and frequency domain. Therefore, many studies [19-23] have used the multilayer wavelet decomposition transform to remove noise. Wavelet transforms can decompose the noise and the signal into different frequency bands, but they do not have the ability to adaptively identify which sub-band is the noise.

The fractal theory proposed by Mandelbrot [24] provides the possibility of analyzing the similarity and correlation between the measured signal and the noise signal. In particular, multifractals $[25,26]$ enable a detailed description of the local characteristics of the signal. Combining the theory of multifractals and detrended fluctuation analysis, Kantelhardt et al. [27] developed multifractal detrended fluctuation analysis (MFDFA). MFDFA has since been widely applied to stock market analysis [28], temperature time series [29], seismic wave signals [30], vibration tomographic diagnosis [31], image processing [32], and voice signal analysis [33]. MFDFA can effectively describe the nonlinear measurement signal, especially the multifractal characteristics of the time series, but the analysis of the time series signal requires a detrending process, which causes pseudofluctuation errors to appear. There are two main reasons for this: one is that the sequence is over- or underfitted due to the uncertainty of the order of the fitted polynomial function; the other is that MFDFA uses a uniform sequence for data segmentation, resulting in sequence segmentation points that are not continuous. To solve these problems, $\mathrm{Li}$ et al. [34] proposed an MFDFA algorithm based on empirical mode decomposition (EMD) and template movement. This algorithm was used to analyze the multifractal characteristics of harmonic signals, resulting in a new method for determining the characteristics of harmonic signals. This method can be used to analyze the similarity between the noise and signal sub-bands given by wavelet analysis, allowing the signal component and noise component to be identified. The noise component can then be filtered out and the signal component reconstructed, thus realizing signal denoising.

In order to separate noise signal and measurement signal, this article proposes an asymmetric two-electrode measuring device, most signal components of one electrode are electrostatic signals, and the other contains most noise signals. A wavelet transform is used to decompose the electrostatic signals and noise signals, and a time-lapse Pearson correlation analysis method is developed to analyze the correlation between the signal wavelet and the noise subbands to confirm which signal components have the most correlation with noise. The result of time-lapse Pearson determines that $d_{3}$ is a noise component, but it is impossible to determine which other components are noise and which are signals. So the EMD-MFDFA algorithm is then used to analyze the similarity between the signal sub-band and the noise sub-band and determine the wavelet sub-bands in which the electrostatic signal is located, and the results show that $a_{4}, a_{5}, d_{4}$, and $d_{5}$ are the signal components. Finally, the noise is filtered and the denoised electrostatic signal is reconstructed with $a_{4}, a_{5}, d_{4}$, and $d_{5}$.

\section{Analysis Method Combining Multifractals and Empirical Mode Decomposition}

\subsection{Generalized Multifractal Detrending Algorithm [27]}

(1) Determine the "contour" of $x_{k}$ as

$$
Y(i)=\sum_{k=1}^{i}\left(x_{k}-\bar{x}\right),
$$

where $\bar{x}=(1 / N) \sum_{k=1}^{N} x_{k}$.

(2) Divide the contour $Y(i)$ into $N_{s}=\operatorname{int}(N / s)$ equallength nonoverlapping segments with a length of $s$. There may be a segment with a length of less than $s$ at the end of the contour. To eliminate the influence of this part, the same process is repeated from the opposite end to obtain $2 N_{s}$ data segments.

(3) Calculate the local trend of each of the $2 N_{s}$ segments through the least-squares fitting of the sequence, and then determine the variance as 


$$
F^{2}(s, v)=\frac{1}{s} \sum_{i=1}^{s}\left\{Y[(v-1) s+i]-y_{v}(i)\right\}^{2}
$$

where each segment is denoted by $v, v=1, \ldots, N_{s}$, and

$F^{2}(s, v)=\frac{1}{s} \sum_{i=1}^{s}\left\{Y\left[N-\left(v-\left|N_{s}\right|\right) s+i\right]-y_{v}(i)\right\}^{2}$,

where $v=N_{s}+1, \ldots, 2 N_{s} . \quad y_{v}(i)$ is the fitting polynomial of the data segment $v$. Linear, quadratic, cubic, or higher-order polynomials can be used in the fitting process (generally called DFA1, DFA2, DFA3, and so on). As the time series is detrended by subtracting the polynomial fitting from the contour, the ability of the different-order DFAs to eliminate the trend of the series is different. In m-order MFDFA, the m-order trend (or, equivalently, the $(m-1)$-order trend in the original sequence) in the contour is eliminated.

(4) Take the average of all line segments to obtain the q-order wave function

$$
F_{q}(s)=\left\{\frac{1}{2 N_{s}} \sum_{v=1}^{2 N_{s}}\left[F^{2}(s, v)\right]^{q / 2}\right\}^{1 / q},
$$

where $F_{q}(s)$ is a q-order function of $s$. As $s$ increases, $F_{q}(s)$ has a power relationship with $s$ :

$$
s^{h(q)} \propto F_{q}(s),
$$

where $h(q)$ is the Hurst index, which is related to the multifractal quality index $\tau(q)$ according to

$$
\tau(q)=q h(q)-1
$$

(5) According to the Legendre transformation, the relationship between the singular index $\alpha$ and the multifractal spectrum $f(\alpha)$ and $h(q)$ is

$$
\begin{aligned}
\alpha & =h(q)+q h^{\prime}(q)=h(q)+q \frac{\mathrm{d} h(q)}{\mathrm{d} q}, \\
f(\alpha) & =q[\alpha-h(q)]+1 .
\end{aligned}
$$

2.2. Empirical Mode Decomposition Algorithm [35, 36]. EMD enables the smoothing of nonstationary signals, with fluctuations and changing trends on different characteristic time scales identified by decomposing the signal. The decomposition result is composed of multiple intrinsic mode functions (IMFs) $c_{1}(t), c_{2}(t), \ldots, c_{n}(t)$ and residuals $r_{n}(t)$, where $r_{n}(t)$ is a monotonic function that is the average trend of the signal.

The EMD process can be described as follows:

$$
x(t)=\sum_{i=0}^{n} c_{i}(t)+r_{n}(t)
$$

2.3. Improved Method Based on EMD-MFDFA. Analyzing the third step of the MFDFA algorithm, it can be seen that the order of the fitting polynomial $y_{v}(i)$ cannot be determined at the initial stage of signal analysis. $y_{v}(i)$ is obtained by curve fitting. If the order is very high, it will take a long time to determine the order, and the determined polynomial may not be optimal. Improper fitting of the volatility trend will introduce erroneous volatility and affect the analysis results. In the EMD result, $r_{n}(t)$ reflects the average trend characteristics of the signal. Using $r_{n}(t)$ instead of $y_{v}(i)$ in the third step of the MFDFA constitutes a new EMDMFDFA algorithm.

Third step: the local variance of each of the $2 N_{s}$ segments is

$$
F^{2}(s, v)=\frac{1}{s} \sum_{i=1}^{s}\left\{Y[(v-1) s+i]-r_{v}(i)\right\}^{2},
$$

where each segment is denoted by $v, v=1, \ldots, N_{s}$, and

$$
F^{2}(s, v)=\frac{1}{s} \sum_{i=1}^{s}\left\{Y\left[N-\left(v-\left|N_{s}\right|\right) s+i\right]-r_{v}(i)\right\}^{2},
$$

where $v=N_{s}+1, \ldots, 2 N_{s}$.

The first, second, and fourth steps of the EMD-MFDFA algorithm are the same as in the MFDFA algorithm. The following research compares the effects of the MFDFA algorithm and the EMD-MFDFA algorithm in multiple analysis applications.

2.4. Time-Lapse Pearson Analysis Method. The Pearson correlation coefficient describes the correlation between two variables at the same time and does not consider the time lag of the change between variables. This paper proposes a Pearson correlation coefficient method with time-lapse correlation analysis, which enables the time-lapse correlation between two variables to be analyzed.

The Pearson correlation coefficient between samples $X$ and $Y$ of a total of $N$ samples is given by

$$
r=\frac{\sum_{i=1}^{N}\left(x_{i}-\bar{x}\right)\left(y_{i}-\bar{y}\right)}{\sqrt{\sum_{i=1}^{N}\left(x_{i}-\bar{x}\right)^{2} \sum_{i=1}^{N}\left(y_{i}-\bar{y}\right)^{2}}}
$$

where $\bar{x}=(1 / N) \sum_{i=1}^{N} x_{i}, \bar{y}=(1 / N) \sum_{i=1}^{N} y_{i}, \quad X=\left[x_{1}, \ldots\right.$, $\left.x_{N-1}, x_{N}\right]$, and $Y=\left[y_{1}, \ldots, y_{i-1}, y_{i}\right]$.

The range of Pearson's correlation coefficient $r$ is $[-1,1]$; $r \mid$ represents the degree of correlation between two variables, with $|r|$ closer to 1 indicating that the correlation between the two variables is higher, and the relationship between them is closer.

The time-lapse Pearson correlation between $X$ and $Y_{j}$ is defined as

$$
r_{j}=\frac{\sum_{i=1}^{N}\left(x_{i}-\bar{x}\right)\left(y_{i+j}-\overline{y_{j}}\right)}{\sqrt{\sum_{i=1}^{N}\left(x_{i}-\bar{x}\right)^{2} \sum_{i=1}^{N}\left(y_{i+j}-\overline{y_{j}}\right)^{2}}}
$$

where $\bar{x}=(1 / N) \sum_{i=1}^{N} x_{i}, \overline{y_{j}}=(1 / N) \sum_{i=1}^{N} y_{i+j}, X=\left[x_{1}, \ldots\right.$, $\left.x_{N-1}, x_{N}\right]$, and $Y_{j}=\left[y_{j}, y_{j+1}, \ldots, y_{i+j-1}, y_{i+j}\right]$.

The time-lapse Pearson correlation matrix is 


$$
R=\left[r_{-j}, r_{-j+1}, \ldots, r_{-1}, r_{0}, r_{1}, \ldots, r_{j-1}, r_{j}\right] .
$$

\section{Signal Acquisition}

The study in [15] describes a number of treatments for weak signal-detection circuit design and noise shielding and finds that the measurement signal is still inevitably subjected to interference by surrounding noise signals. Obviously, in addition to the necessary hardware protection to reduce noise interference, noise removal is required. First, it is necessary to understand the morphological characteristics of the noise signal obtained by the detection circuit, and to distinguish the noise components in the measurement signal. The noise in the measurement signal can then be effectively removed.

3.1. Asymmetric Two-Electrode Measuring Device. To remove noise, an asymmetric two-electrode measurement unit is developed. One electrode is the noise collection terminal, which mainly collects noise signals in the detection environment, and the other electrode is the signal collection terminal, which mainly collects noise-containing microelectric signals. According to the positive correlation between the induction signal and the size of the electrode [6], it can be assumed that the induction signal obtained by the noise-collecting electrode is much smaller than the induction signal measured by the measuring electrode and that noise is the main component. The noise-collecting electrode is much smaller than the measuring electrode, having a length of $20-30 \mathrm{~mm}$ and a diameter of 1-2 $\mathrm{mm}$. The measuring electrode has a length of $400 \mathrm{~mm}$ and a diameter of $10 \mathrm{~mm}$. The structure of the measuring device is shown in Figure 1.

3.2. Acquisition of Measurement Signal. Figures 2(a) and 2(b) show the dust microelectric signal collected by the measuring device. It can be seen that the signal induced on the measuring electrode by the movement of the dust is much larger than the signal collected by the noise-collecting electrode. In the measurement signal recorded by the noise electrode, the effective induction signal is almost completely submerged in the noise.

\section{Characteristic Analysis of Measurement Signal and Measurement Noise Signal}

Figure 2 illustrates that the measurement signal exhibits nonlinear and random characteristics, as does the noise in the measurement environment. To effectively filter the noise from the measurement signal, the measurement noise needs to be identified. First, wavelet decomposition is applied to the signal recorded by the measuring electrode to obtain the frequency components in different frequency ranges. Timelapse Pearson analysis is then performed on the different frequency components and noise signals to identify those components that have the strongest correlation with the noise. Using the MFDFA algorithm and the improved EMD-

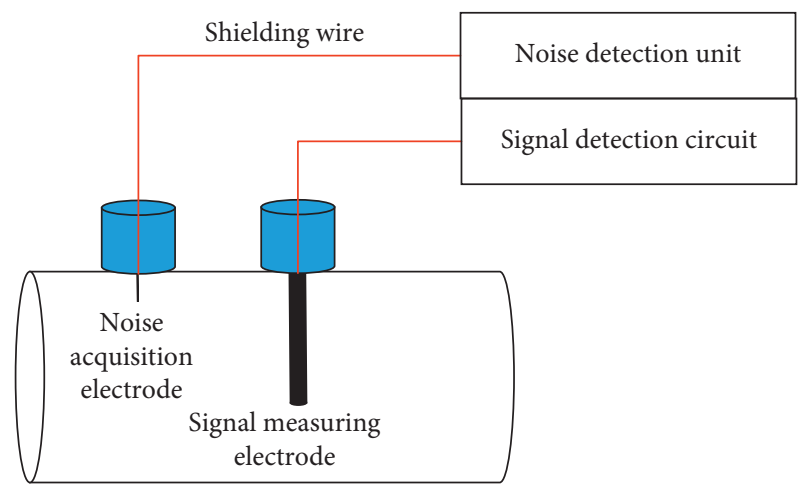

FIGURE 1: Schematic figure of the structure of the measuring device.

MFDFA algorithm, the similarity between the different frequency bands of the signal and the measurement noise is then analyzed, and the frequency components closest to the collected noise are removed. This analysis makes it possible to determine the characteristics of the noise that affect the measurement signal, and to obtain the noise signal as the basis for noise filtering. The architecture is shown in Figure 3.

\subsection{Wavelet Analysis of Measurement Signals}

4.1.1. db24 Wavelet Decomposition. The application of the db24 wavelet with five-layer decomposition to the measured signal shown in Figure 2(a) gives the signal waveforms of each sub-band, as shown in Figure 4.

The frequency range of each sub-band is listed in Table 1. Analysis shows that each decomposed sub-band contains corresponding frequency components, and the signal amplitudes of the high-frequency signal parts $d_{1}, d_{2}$, and $d_{3}$ are small, but not negligible.

\subsection{Time-Lapse Pearson Correlation Analysis of the Measured Signal Sub-Band and the Noise Signal}

4.2.1. Analysis of Time-Lapse Pearson Correlation between the Measured Signal Sub-Band and the Noise Signal. Figure 5 shows the result of direct analysis of the correlation between the measured signal sub-band and the noise signal for about 10s (5000 data points). The focus is whether the change in noise occurs first, or whether the detection signal leads.

From Figure 5, the following points can be identified: (1) the time-lapse correlation coefficient between component $d_{3}$ and the noise is greater than 0.6 , indicating a strong correlation between the two; (2) the two signals appear to change simultaneously (only 1-point difference, $0.002 \mathrm{~s}$ ), and the frequency range of $d_{3}$ is $29.75-59.5 \mathrm{~Hz}$.

4.2.2. Wavelet Sub-Band Decomposition of Noise Signal. By analyzing the time-lapse Pearson correlation between the wavelet sub-band of the measured signal and the measured noise, it is clear that there is a strong correlation between $d_{3}$ and the noise, although the two are not completely 


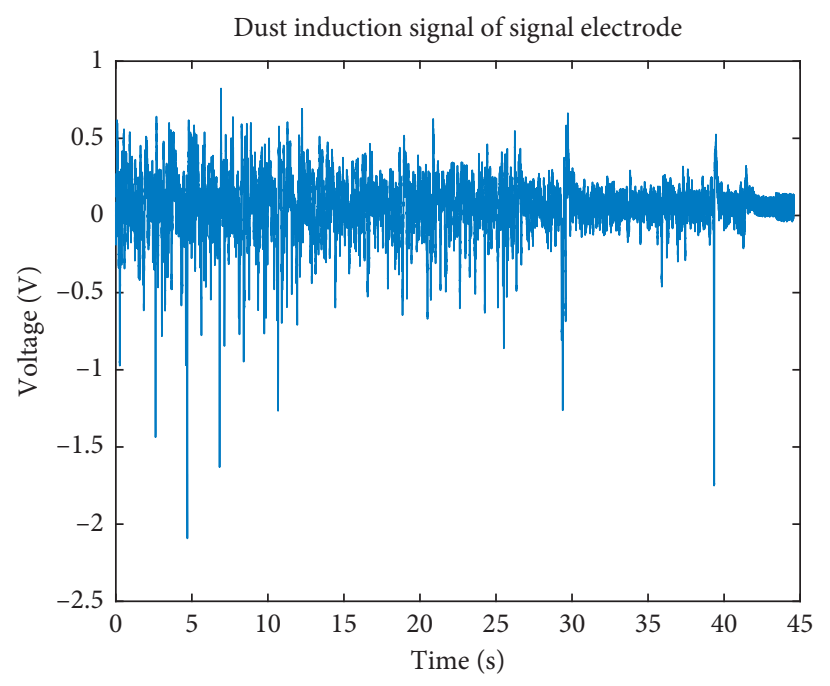

(a)

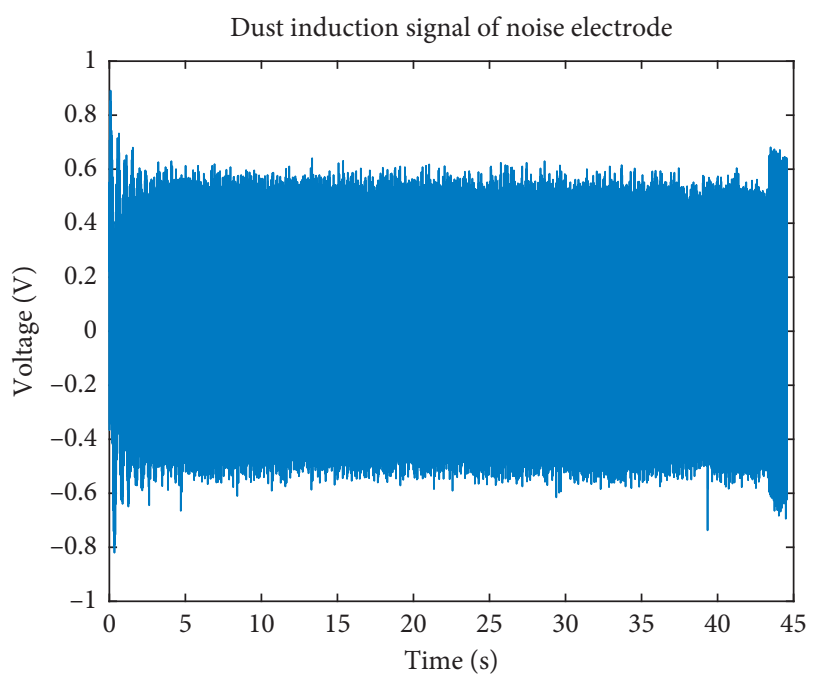

(b)

Figure 2: Acquisition of measurement signal. (a) Signal of signal-measuring electrode. (b) Signal of noise-measuring electrode.

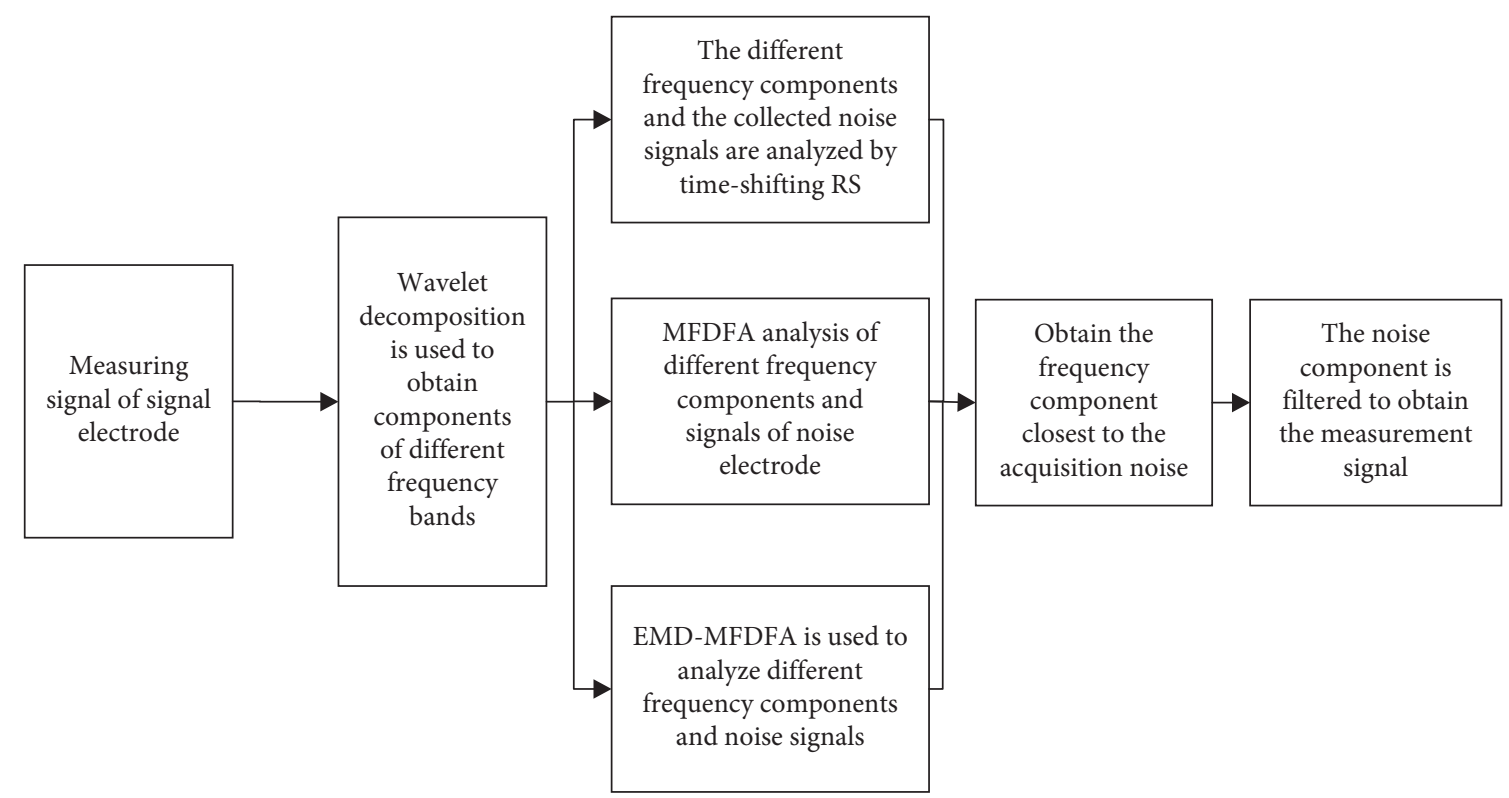

FIGURE 3: Architecture diagram of feature analysis.

correlated. The noise contains a strong $50 \mathrm{~Hz}$ component, which masks the correlation between other components and the noise. First, the same method as for measurement signal analysis is used to decompose the noise signal into its subbands; the wavelet sub-band ranges of the noise are the same as in Table 1.

The time-lapse Pearson correlation between the measured signal wavelet sub-bands and the noise wavelet subbands is presented in Table 2 . The following correlations can be identified:

(1) nos $\_a_{4}-a_{4}$ and nos $\_a_{5}-a_{5}$ are highly correlated and exhibit the characteristics of simultaneous changes
(2) nos $d_{3}-d_{3}$ indicates that, given the strong correlation between $d_{3}$ and the overall noise signal, component $d_{3}$ can be considered as a noise signal

(3) nos $d_{1}-d_{1}$, nos $a_{3}-a_{3}$, and nos $d_{5}-d_{5}$ exhibit a certain correlation, but it is impossible to directly determine which is the noise component and which is the signal

4.3. Multifractal Characteristic Analysis of Signal and Noise. The MFDFA algorithm is used to study the multifractal characteristics of the measured signal and the measured noise, which is judged as follows. 

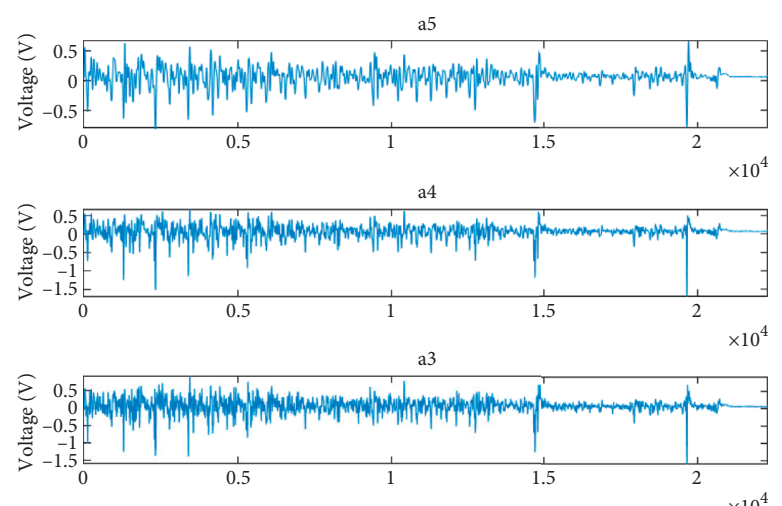

$\times 10^{4}$

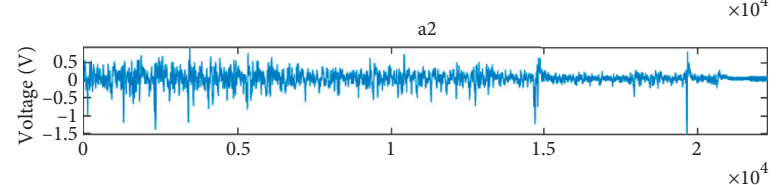

$\times 10^{4}$

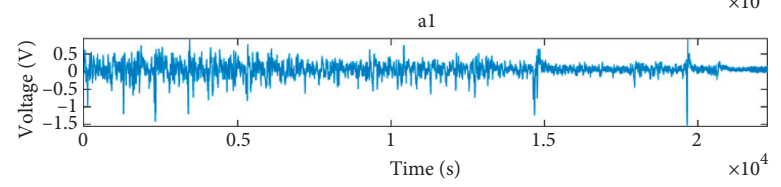

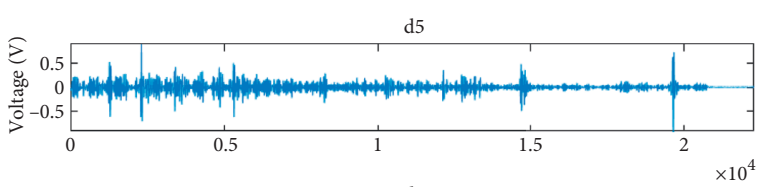

$\mathrm{d} 4$

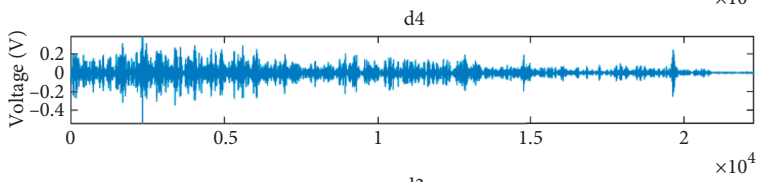

d3
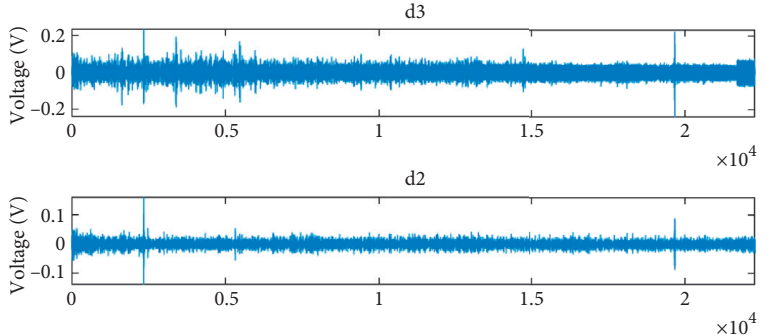

d1

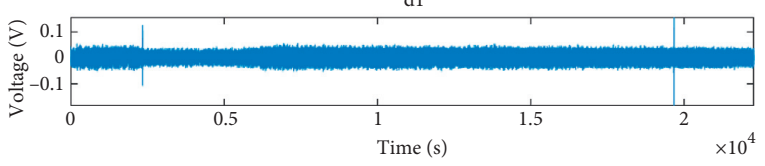

FIGURE 4: Wavelet sub-band waveforms of measuring electrode's signal.

TABLE 1: Wavelet sub-band frequency range of detection signal.

\begin{tabular}{lc}
\hline Noise signal decomposition layer & Sub-band frequency \\
\hline$a_{5}$ & $0-7.4375 \mathrm{~Hz}$ \\
$a_{4}$ & $0-14.875 \mathrm{~Hz}$ \\
$a_{3}$ & $0-29.75 \mathrm{~Hz}$ \\
$a_{2}$ & $0-59.5 \mathrm{~Hz}$ \\
$a_{1}$ & $0-119 \mathrm{~Hz}$ \\
$d_{5}$ & $7.4375-15.625 \mathrm{~Hz}$ \\
$d_{4}$ & $14.875-29.75 \mathrm{~Hz}$ \\
$d_{3}$ & $29.75-59.5 \mathrm{~Hz}$ \\
$d_{2}$ & $59.5-119 \mathrm{~Hz}$ \\
$d_{1}$ & $119-238 \mathrm{~Hz}$ \\
\hline
\end{tabular}

Relationship between $q$ and $h(q)$ : for $q=2$, the Hurst exponent $h(q)$ has the following properties [37]: when $h(q)=0.5$, the signal sequence has no correlation; when $h(q)>0.5$, the signal sequence has a positive long-range correlation, indicating that the time series is positively correlated; when $h(q)<0.5$, the time series is negatively correlated. If $q$ is not related to $h(q)$, the signal is monofractal; if $q$ is related to $h(q)$, the signal is multifractal.

Relationship between $q$ and $\tau(q)$ : if $\tau(q)$ is a straight line, then the signal is simplex; if $q$ and $\tau(q)$ are nonlinear, then the signal is multifractal.

Relationship between $\alpha$ and $f(\alpha)$ : if $f(\alpha)$ is a constant, the signal is a single-shaped signal; if the curve between $\alpha$ and $f(\alpha)$ has a single-peak bell shape, the signal is multifractal.

MFDFA analysis was applied to the measured signal and the measured noise signal, and the results are shown in Figure 6. Both $q-\tau(q)$ and $\alpha-f(\alpha)$ indicate that the measured signal and measured noise do not have multifractal characteristics.
The multifractal features of the signal are submerged by noise pollution. Next, by analyzing the multifractal features of the measured signal and measured noise on each wavelet sub-band, it is possible to determine which sub-bands have noise signals as their main components and which are mainly measured signals.

4.3.1. Analysis of the Detrending Ability of MFDFA and EMD-MFDFA Algorithms. The $\log (F q)-\log (s)$ curve of the wavelet sub-band $d$ component of the signal was obtained using MFDFA and EMD-MFDFA. The detrending polynomial of the MFDFA algorithm is linear, and the EMD-MFDFA detrending term is the residual $r_{n}(t)$ of EMD. The results are shown in Figure 7. From the value of $F q$ in Figure 7, we find that the absolute value of the result obtained by EMD-MFDFA and its numerical range are smaller than those obtained by MFDFA. This shows that EMDMFDFA has stronger detrending ability than MFDFA.

\subsubsection{Analysis of MFDFA and EMD-MFDFA Characteristics: Wavelet Sub-Bands of Measurement Signals and Wavelet Sub-Bands of Noise Signals}

(1) $q-h(q)$ Characteristic Analysis. The wavelet sub-bands of the measured signal and the measured noise obtained from MFDFA and EMD-MFDFA are now analyzed. The results are shown in Figure 8. For $q=2$, the Hurst exponent $h(q)$ of each wavelet sub-band component of the measured signal and noise and its long-range correlation are presented in Tables 3 and 4. Analysis of the MFDFA and EMD-MFDFA algorithms shows that EMD-MFDFA can effectively eliminate the influence of the trend item, so that the trend characteristic of the affected component is 

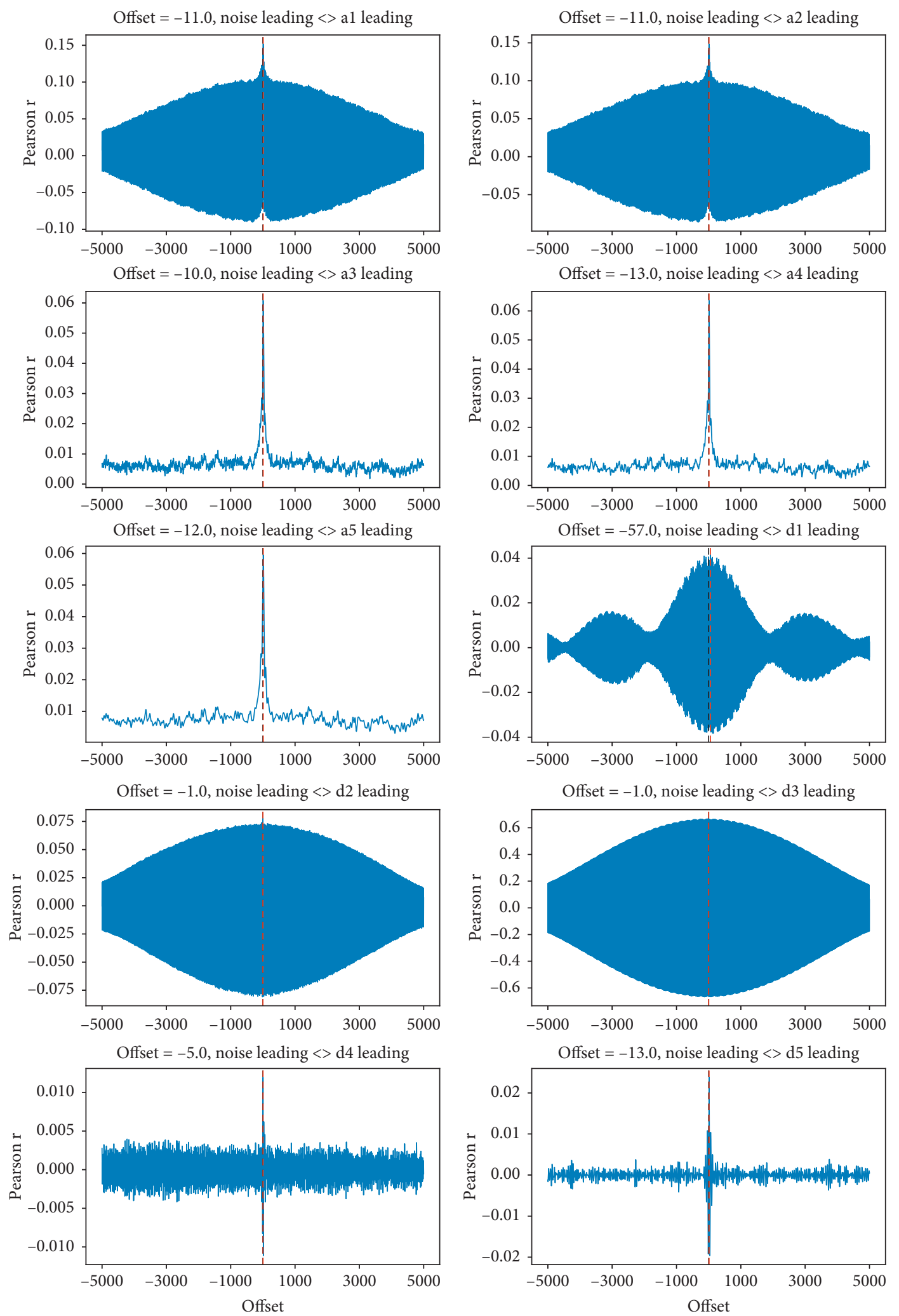

FIgURE 5: Correlation diagram of each sub-band signal and noise signal.

eliminated. This highlights the multifractal characteristics of the component. By comparing the $q-\tau(q)$ values of signal components $a_{1}$ and $a_{3}$ in Figure 8 , it can be seen that EMD-MFDFA highlights the multifractal characteristics of the measured signal components. EMD-MFDFA gives smaller $k$ values for acoustic components $a_{2}, d_{2}$, and $d_{3}$ than MFDFA, which leads to the fact that the measured signal does not have multifractal characteristics. 
TABle 2: Time-lapse Pearson correlation.

\begin{tabular}{lcc}
\hline Analysis object & Max value & $\begin{array}{c}\text { Offset time (positive value indicates that noise component leads; } \\
\text { negative value indicates that signal component leads) }\end{array}$ \\
\hline nos_ $a_{1}-a_{1}$ & 0.160 & 0 \\
nos_ $a_{2}-a_{2}$ & 0.160 & 0 \\
nos_ $a_{3}-a_{3}$ & 0.450 & 0 \\
nos_ $a_{4}-a_{4}$ & 0.667 & 0 \\
nos_ $a_{5}-a_{5}$ & 0.710 & 2 \\
nos_d $d_{1}-d_{1}$ & 0.497 & -57 \\
nos_d $d_{2}-d_{2}$ & 0.367 & 0 \\
nos_ $d_{3}-d_{3}$ & 0.694 & 0 \\
nos_d $d_{4}-d_{4}$ & 0.112 & 0 \\
nos_ $d_{5}-d_{5}$ & 0.530 & 0 \\
\hline
\end{tabular}
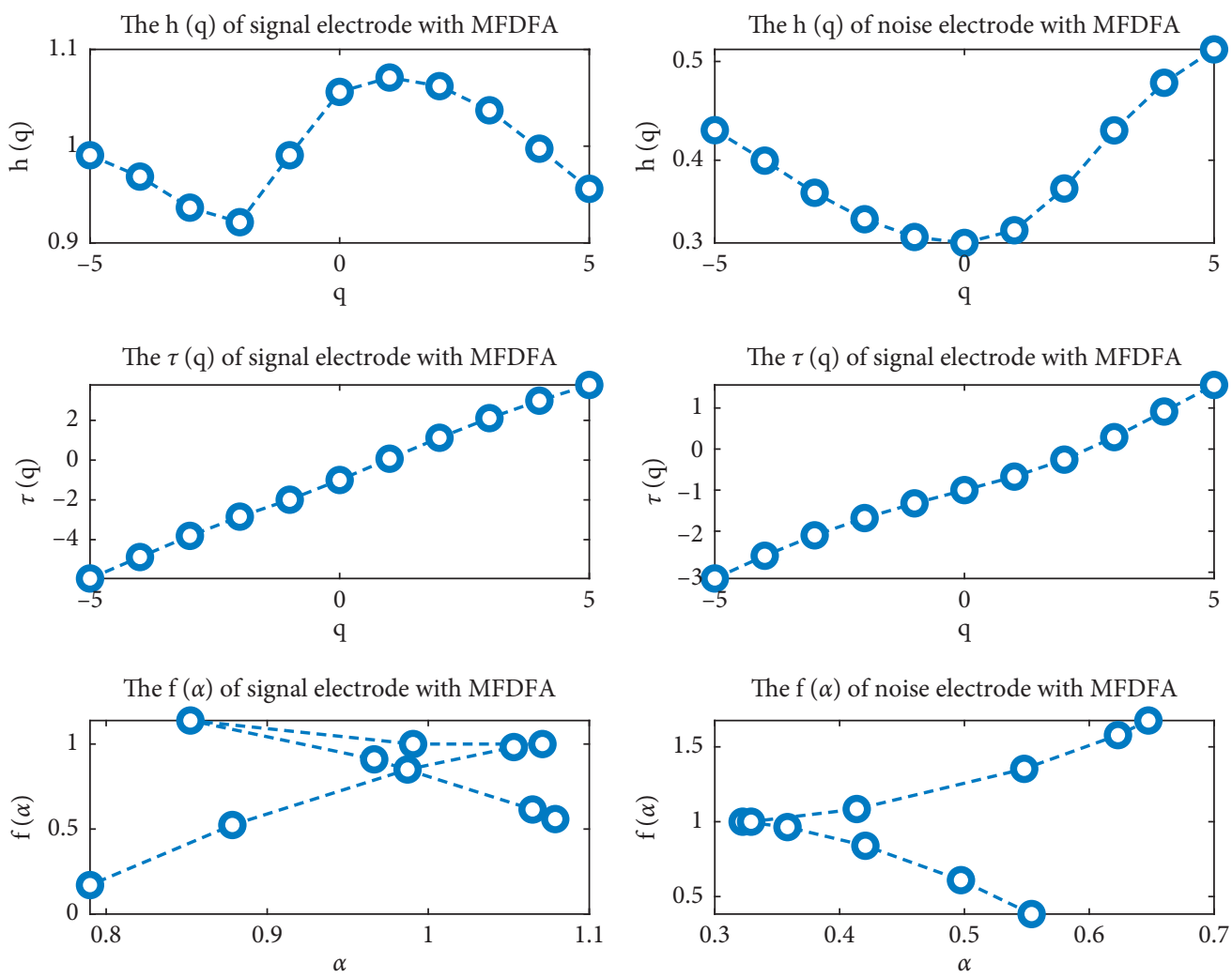

FIgURE 6: Multifractal characteristic figures of measured signal/noise with MFDFA.

(2) $q-\tau(q)$ Characteristic Analysis. Next, MFDFA and EMD-MFDFA were used to obtain the wavelet sub-bands of the measured signal and the measured noise, and the linearity $R$ between $q$ and $\tau(q)$ was calculated. The results are presented in Tables 5 and 6 . Comparing the relationship between $R$ and $q-\tau(q)$, it is apparent that values of $\tau(q)$ greater than 0.96 indicate multifractal characteristics. EMDMFDFA effectively reduces the trend characteristics of components and highlights their fractal characteristics.

(3) $\alpha-f(\alpha)$ Characteristic Analysis. MFDFA and EMDMFDFA were then used to obtain the wavelet sub-band signals of the measured signal and the measured noise, and the $\alpha-f(\alpha)$ waveforms were calculated. The waveforms are shown in Figure 9, and the fractal feature judgments are presented in Tables 7 and 8 .
(4). Determination of Signal Wavelet Sub-Band. The correlation, average error, and standard deviation are now used to evaluate the differences among the EMD-MFDFA analysis results of each sub-band using $h(q), \tau(q)$, and $f(\alpha)$. The results are presented in Tables $9-11$. Combining the results of Figures 8-10, it can be seen that the multifractal features of $a_{4}-$ nos_a $a_{4}, a_{5}-$ nos_$_{-} a_{5}, d_{4}-$ nos_$_{-} d_{4}$, and $d_{5}-$ nos_$_{-} d_{5}$ have very high consistency. These components are mainly concentrated at low frequencies, so $a_{4}, a_{5}, d_{4}$, and $d_{5}$ can be considered as real signal components of the measured signal.

\section{Signal Noise Filtering}

Combining the time-lapse Pearson analysis results and the analysis results using the EMD-MFDFA algorithm, it can be seen that component $d_{3}$ is highly correlated in the 

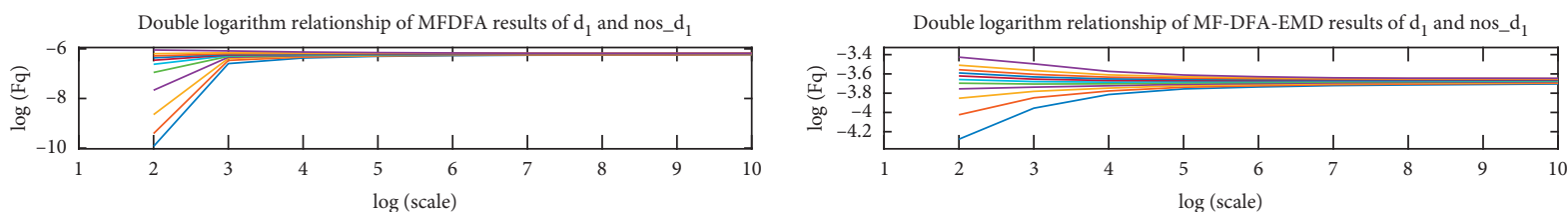

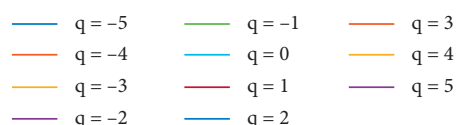

Double logarithm relationship of MFDFA results of $\mathrm{d}_{2}$ and nos_d
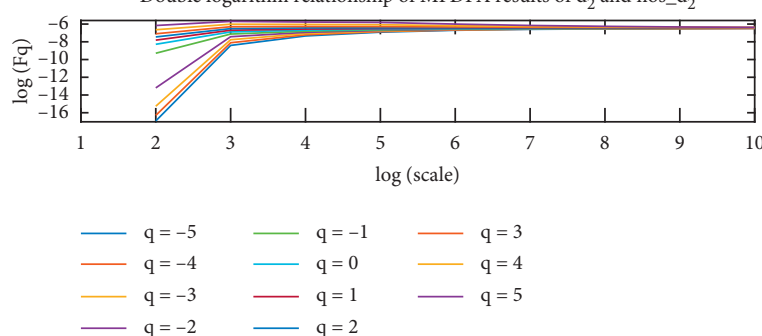

Double logarithm relationship of MFDFA results of $\mathrm{d}_{3}$ and nos_d

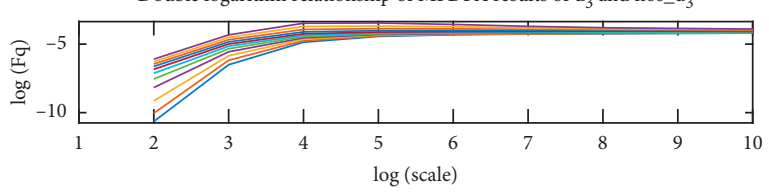

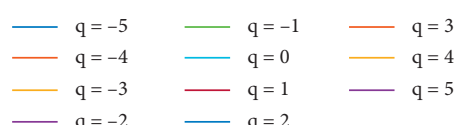

Double logarithm relationship of MF-DFA-EMD results of $\mathrm{d}_{2}$ and nos_ $\mathrm{d}_{2}$
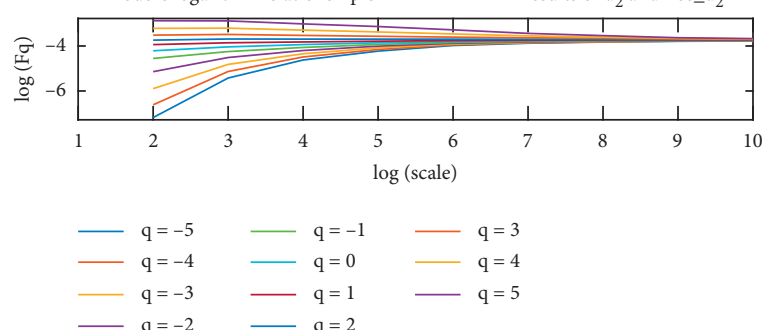

Double logarithm relationship of MF-DFA-EMD results of $d_{3}$ and nos_d $d_{3}$
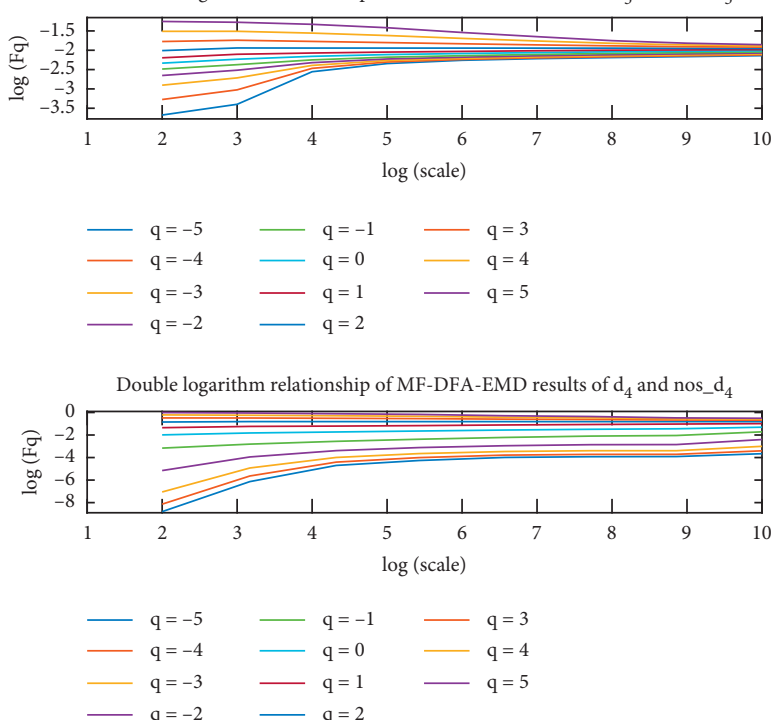

Double logarithm relationship of MF-DFA-EMD results of $\mathrm{d}_{5}$ and nos_d $\mathrm{d}_{5}$
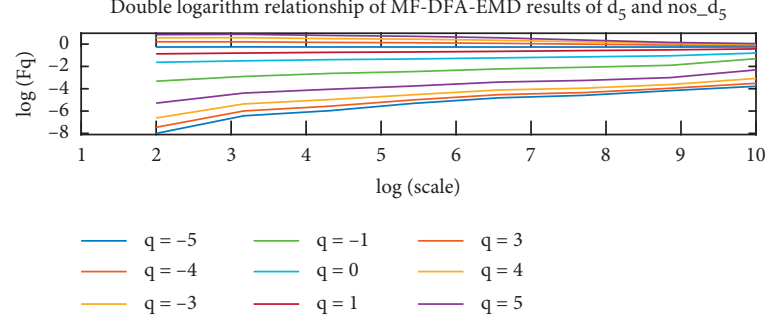
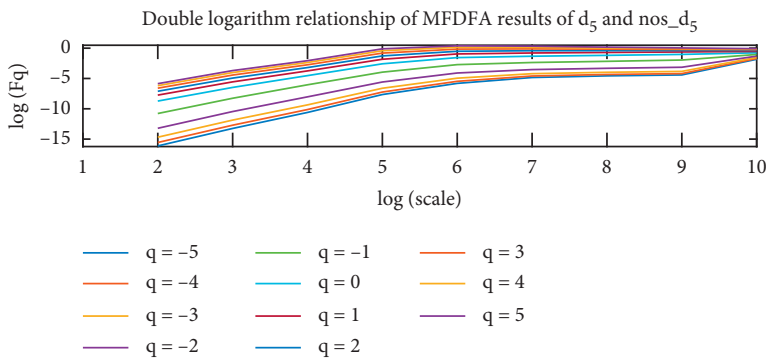

$\longrightarrow q=-2 \quad q=2$

FiguRE 7: MFDFA and EMD-MFDFA detrending capability curves. 

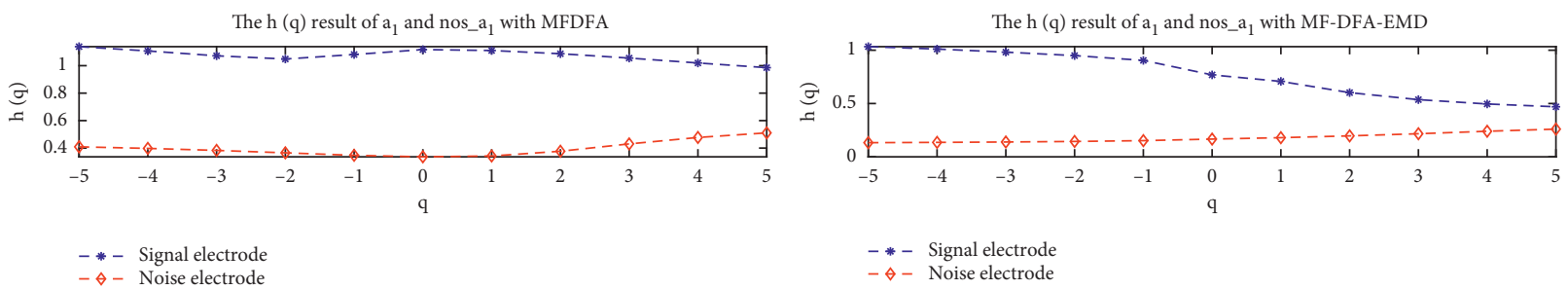

- *- Signal electrode
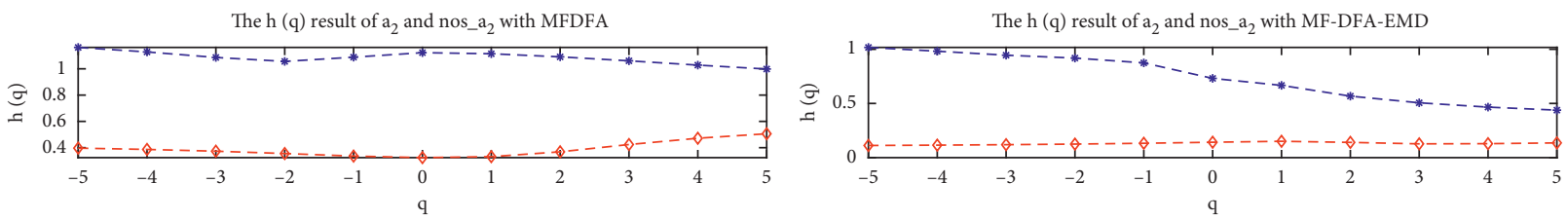

$-*-$ Signal electrode
$-\diamond-$ Noise electrode

- * - Signal electrode

$-\diamond-$ Noise electrode

The $h(q)$ result of $a_{3}$ and nos_ $a_{3}$ with MFDFA
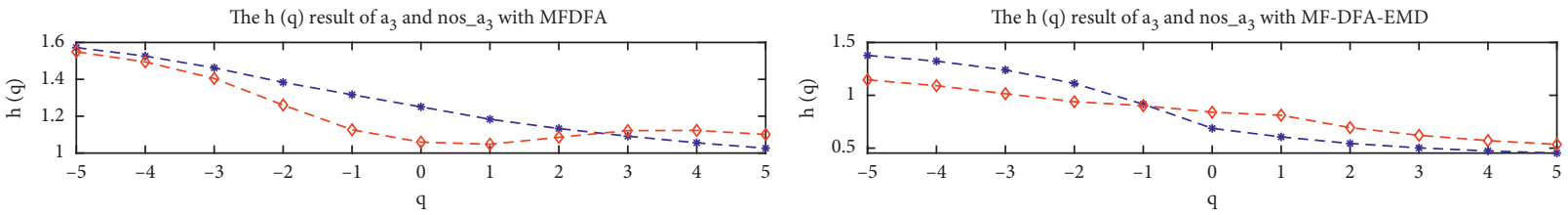

- * - Signal electrode $-\diamond-$ Noise electrode

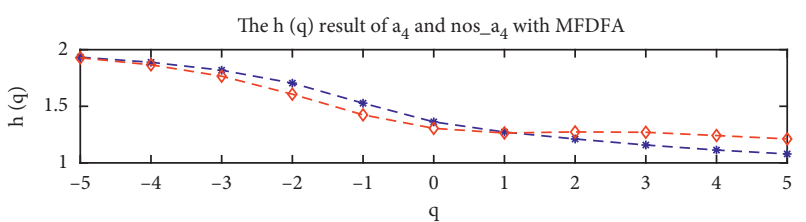

- * - Signal electrode $-\diamond-$ Noise electrode

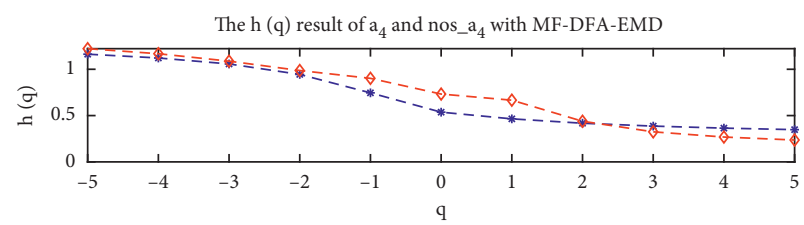

* - Signal electrode

- * - Signal electrode $-\diamond-$ Noise electrode
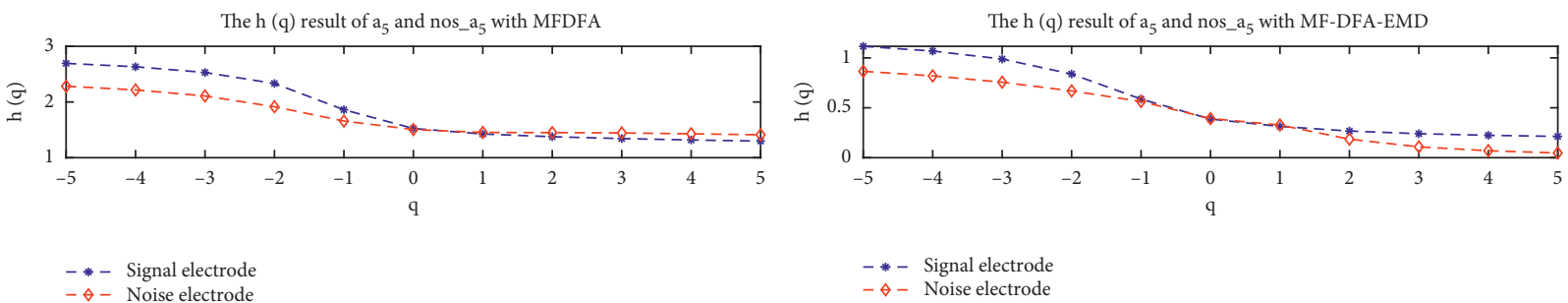

(a)

Figure 8: Continued. 

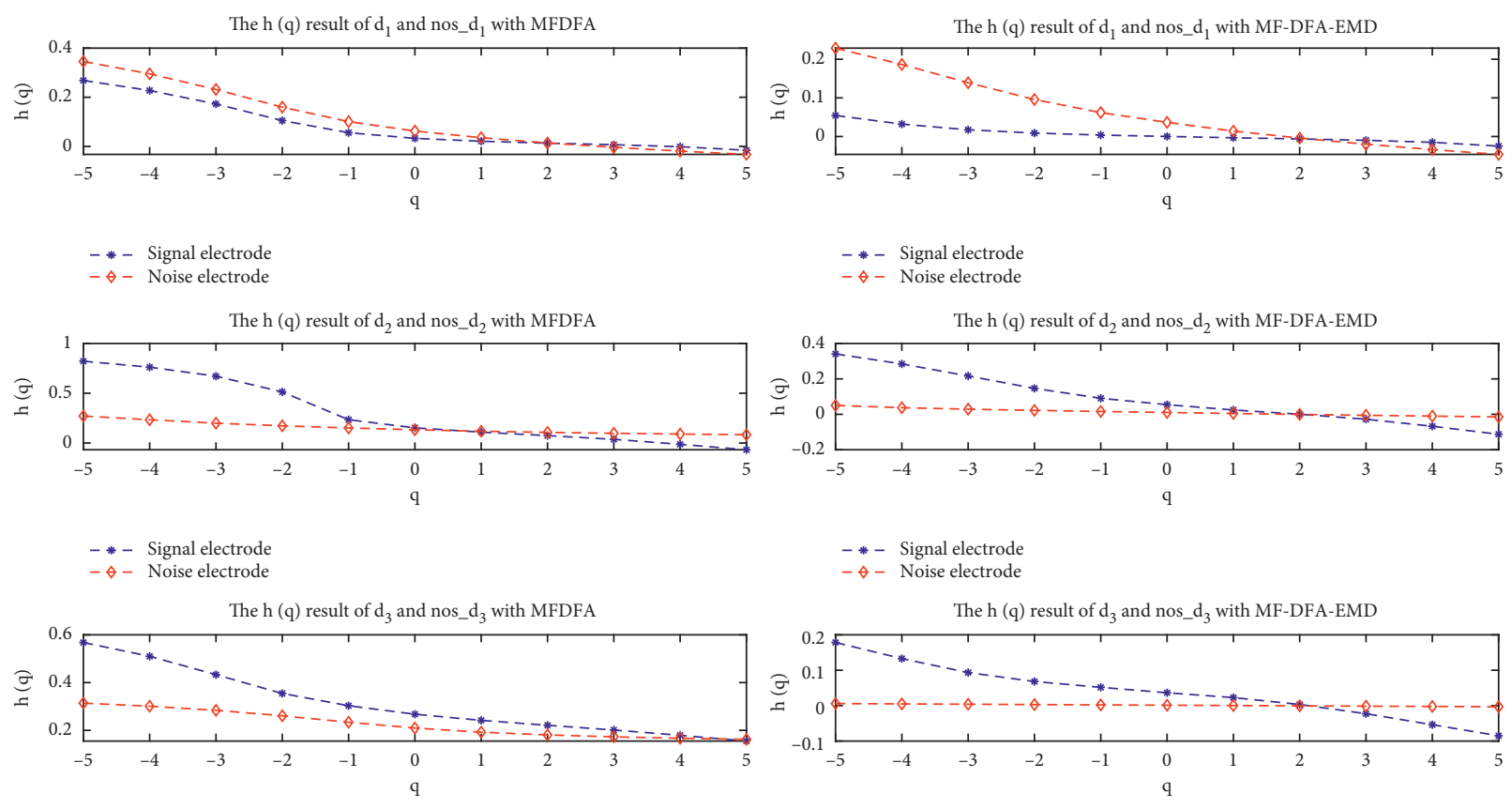

- * - Signal electrode

$-*$ Signal electrode
$-\diamond-$ Noise electrode
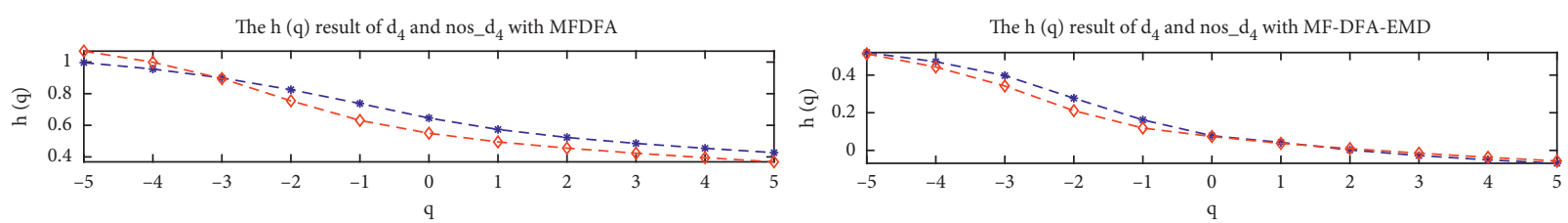

$\begin{array}{ll}-*- & \text { Signal electrode } \\ -\diamond-\text { Noise electrode }\end{array}$

$$
\begin{aligned}
& -*-\text { Signal electrode } \\
& -\diamond-\text { Noise electrode }
\end{aligned}
$$
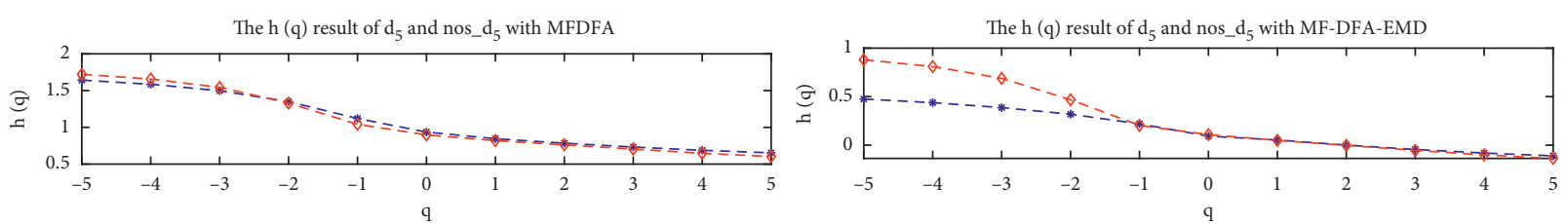

$$
\begin{aligned}
& -* \text { Signal electrode } \\
& -\diamond-\text { Noise electrode }
\end{aligned}
$$$$
\begin{array}{ll}
-*- & \text { Signal electrode } \\
-\diamond-\text { Noise electrode }
\end{array}
$$

(b)

FIGURE 8: MFDFA and EMD-MFDFA $q-h(q)$ curves of each sub-band of the measured signal and measured noise: (a) MFDFA; (b) EMD-MFDFA.

\begin{tabular}{|c|c|c|c|c|c|c|c|c|c|c|}
\hline & $a_{1}$ & $a_{2}$ & $a_{3}$ & $a_{4}$ & $a_{5}$ & $d_{1}$ & $d_{2}$ & $d_{3}$ & $d_{4}$ & $d_{5}$ \\
\hline MFDFA & 1.087 & 1.091 & 1.133 & 1.211 & 1.376 & 0.013 & 0.074 & 0.221 & 0.523 & 0.784 \\
\hline $\begin{array}{l}\text { MFDFA- } \\
\text { EMD }\end{array}$ & 0.602 & 0.567 & 0.544 & 0.417 & 0.266 & -0.006 & 0 & 0.003 & 0.002 & 0 \\
\hline $\begin{array}{l}\text { Relevance } \\
\text { judgment }\end{array}$ & $\begin{array}{l}\text { Negative } \\
\text { correlation }\end{array}$ & $\begin{array}{l}\text { Negative } \\
\text { correlation }\end{array}$ & $\begin{array}{l}\text { Negative } \\
\text { correlation }\end{array}$ & $\begin{array}{c}\text { Positive } \\
\text { correlation }\end{array}$ & $\begin{array}{c}\text { Positive } \\
\text { correlation }\end{array}$ & $\begin{array}{c}\text { Positive } \\
\text { correlation }\end{array}$ & $\begin{array}{c}\text { Positive } \\
\text { correlation }\end{array}$ & $\begin{array}{c}\text { Positive } \\
\text { correlation }\end{array}$ & $\begin{array}{c}\text { Positive } \\
\text { correlation }\end{array}$ & $\begin{array}{l}\text { Positive } \\
\text { correlation }\end{array}$ \\
\hline
\end{tabular}

TABLE 3: For $q=2$, the Hurst exponent of each sub-band component of the measured signal $h(q)$.

TABLE 4: For $q=2$, the Hurst exponent of each sub-band component of measured noise $h(q)$.

\begin{tabular}{lcccccccccc}
\hline & nos $-a_{1}$ & nos $-a_{2}$ & nos $-a_{3}$ & nos $-a_{4}$ & nos $-a_{5}$ & nos $-d_{1}$ & nos $-d_{2}$ & nos $-d_{3}$ & nos $-d_{4}$ & nos $-d_{5}$ \\
\hline MFDFA & 0.377 & 0.369 & 1.086 & 1.274 & 1.450 & 0.014 & 0.107 & 0.181 & 0.455 & 0.762 \\
EMD- & 0.197 & 0.141 & 0.694 & 0.439 & 0.185 & -0.004 & 0 & 0 & 0.009 & -0.002 \\
MFDFA & Positive & Positive & Negative & Positive & Positive & Positive & Positive & $\begin{array}{c}\text { Positive } \\
\text { Relevance }\end{array}$ & $\begin{array}{c}\text { Positive } \\
\text { Positive }\end{array}$ \\
judgment & correlation & correlation & correlation & correlation & correlation & correlation & correlation & correlation correlation correlation \\
\hline
\end{tabular}




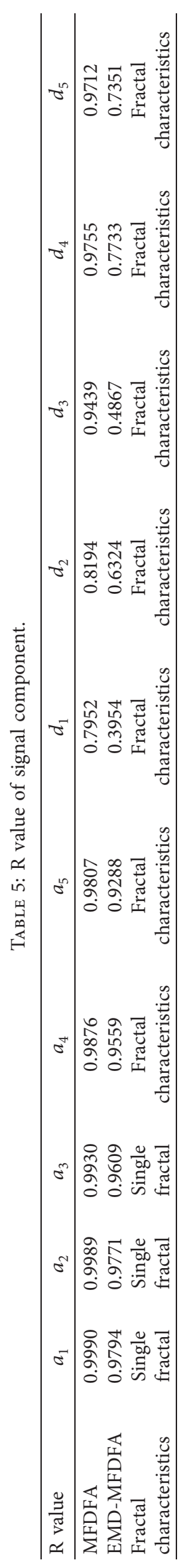




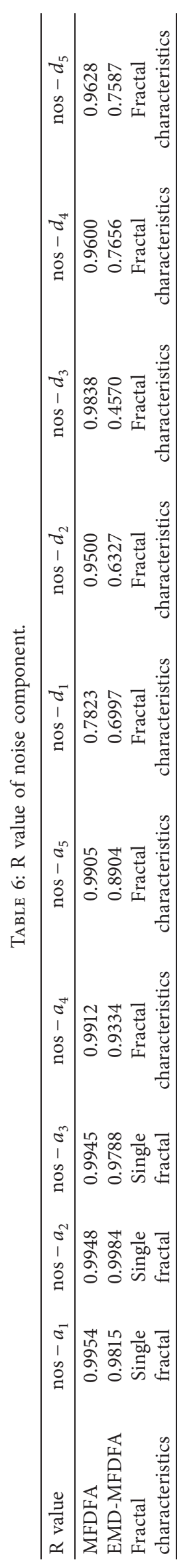



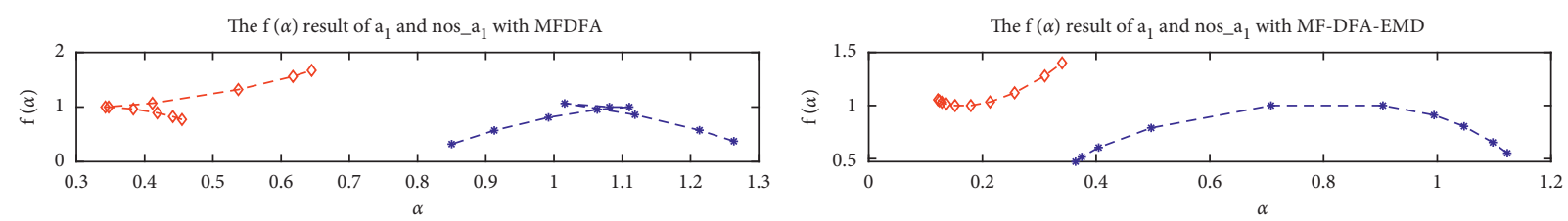

$\begin{array}{ll}-*- & \text { Signal electrode } \\ -\diamond- & \text { Noise electrode }\end{array}$

$$
\begin{array}{ll}
-*- & \text { Signal electrode } \\
-\diamond- & \text { Noise electrode }
\end{array}
$$
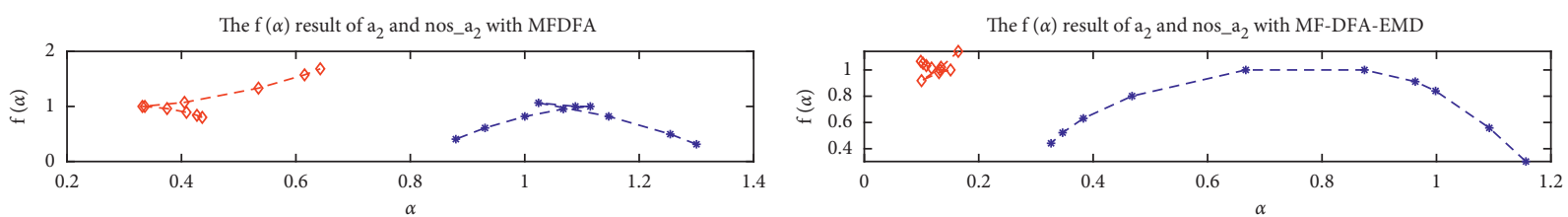

- * - Signal electrode

$-\diamond-$ Noise electrode

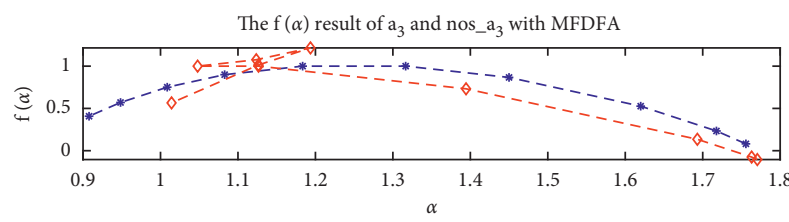

- * - Signal electrode $-\diamond-$ Noise electrode

*- Signal electrode $-\diamond-$ Noise electrode
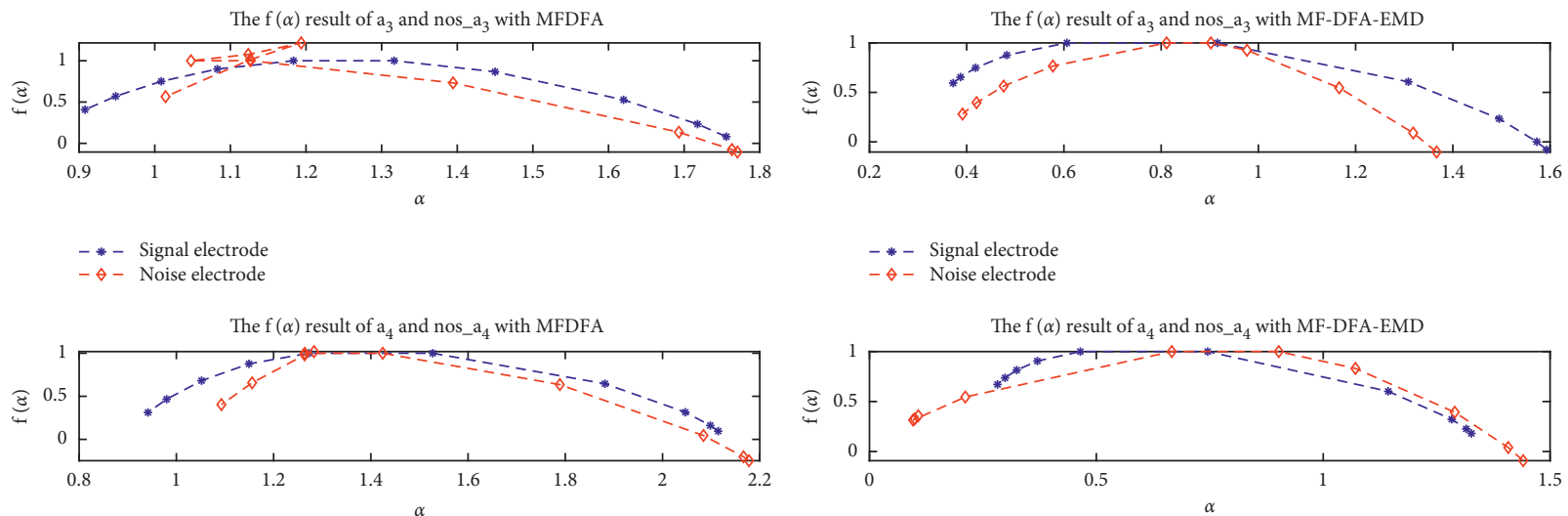

- * - Signal electrode

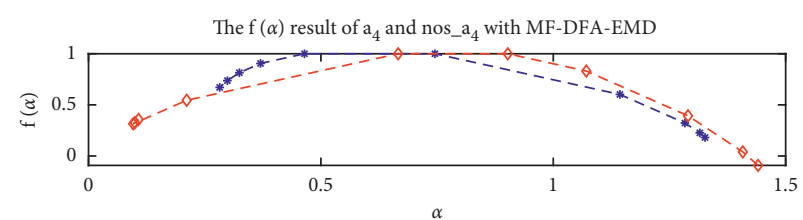

- * - Signal electrode - * - Signal electrode
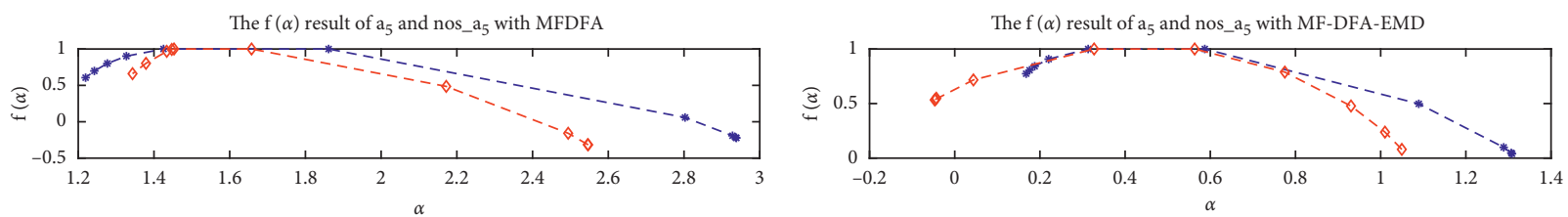

- * - Signal electrode $\begin{array}{ll}-*- & \text { Signal electrode } \\ -\diamond- & \text { Noise electrode }\end{array}$

(a)

FIgURE 9: Continued. 

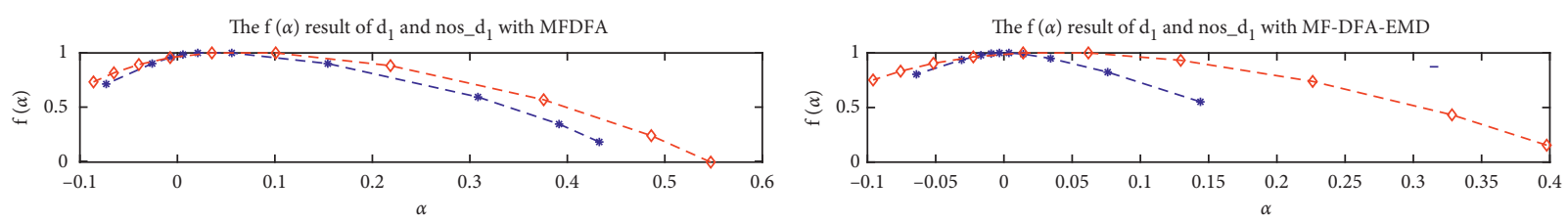

$$
\begin{aligned}
& -*-\text { Signal electrode } \\
& -\diamond-\text { Noise electrode }
\end{aligned}
$$

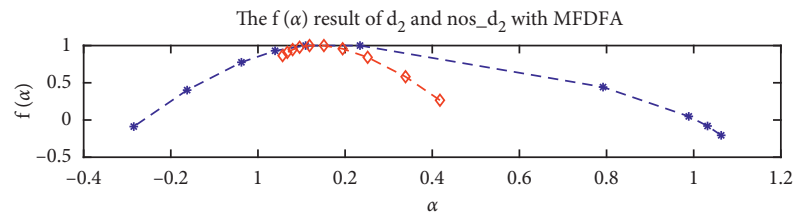

$$
\begin{aligned}
& -*-\text { Signal electrode } \\
& -\diamond-\text { Noise electrode }
\end{aligned}
$$

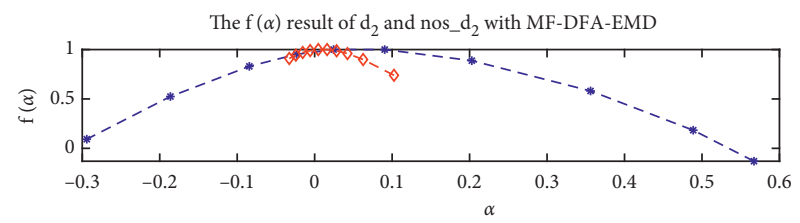

$$
\begin{aligned}
& -*-\text { Signal electrode } \\
& -\diamond-\text { Noise electrode }
\end{aligned}
$$

$-*-$ Signal electrode
$-\diamond-$ Noise electrode
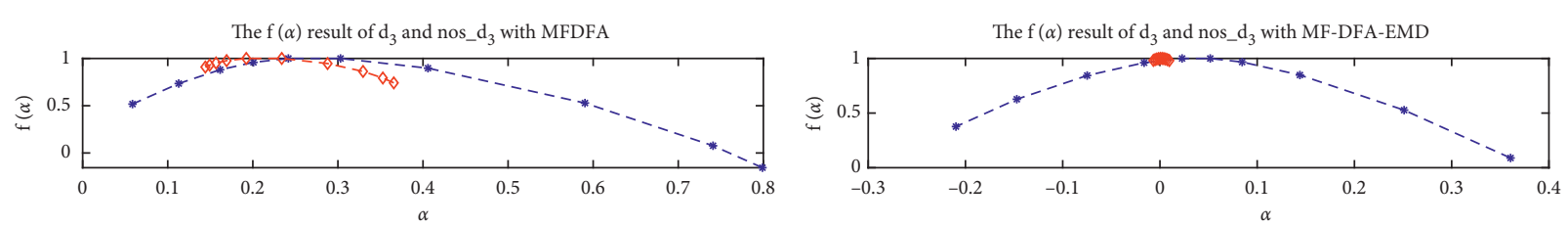

$$
\text { - * - Signal electrode }
$$

- * - Signal electrode

$-\diamond-$ Noise electrode
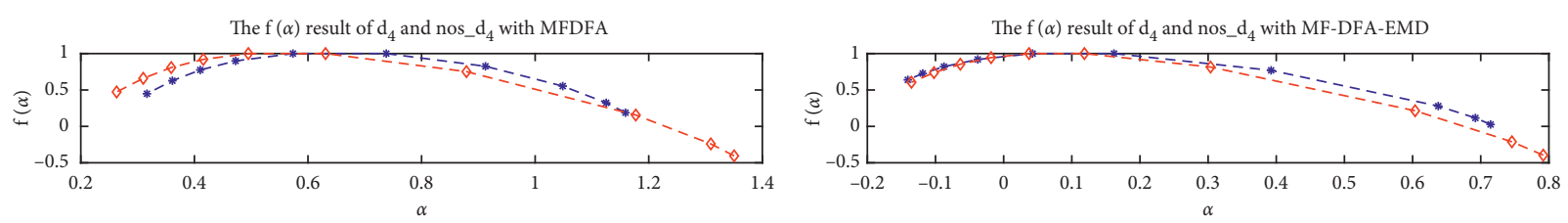

$$
\begin{aligned}
& -*-\text { Signal electrode } \\
& -\diamond-\text { Noise electrode }
\end{aligned}
$$

$\begin{array}{ll}-*- & \text { Signal electrode } \\ -\diamond- & \text { Noise electrode }\end{array}$
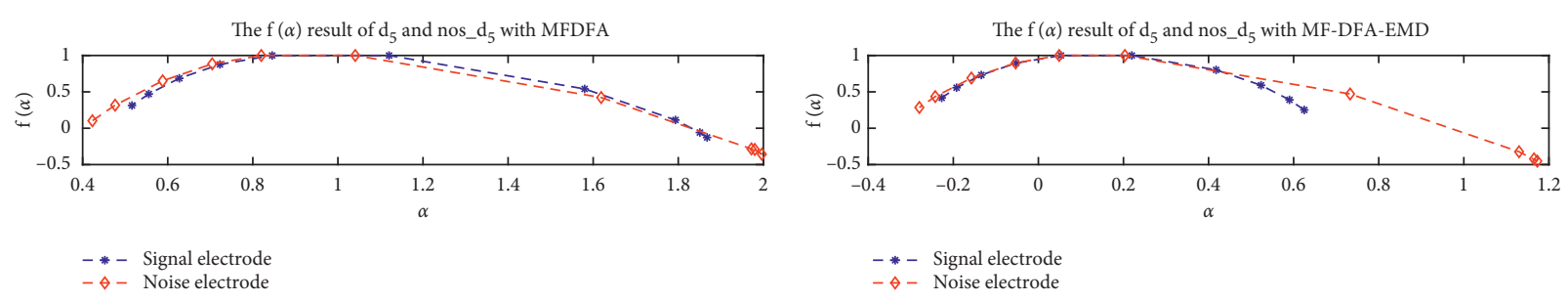

\begin{tabular}{|c|c|c|c|c|c|c|c|c|c|c|}
\hline & $a_{1}$ & $a_{2}$ & $a_{3}$ & $a_{4}$ & $a_{5}$ & $d_{1}$ & $d_{2}$ & $d_{3}$ & $d_{4}$ & $d_{5}$ \\
\hline MFDFA & $\begin{array}{l}\text { Non- } \\
\text { bell } \\
\text { shaped }\end{array}$ & $\begin{array}{c}\text { Non- } \\
\text { bell } \\
\text { shaped }\end{array}$ & $\begin{array}{c}\text { Non- } \\
\text { bell } \\
\text { shaped }\end{array}$ & Bell shaped & Bell shaped & Bell shaped & Bell shaped & Bell shaped & Bell shaped & Bell shaped \\
\hline $\begin{array}{l}\text { EMD- } \\
\text { MFDFA }\end{array}$ & $\begin{array}{c}\text { Non- } \\
\text { bell } \\
\text { shaped }\end{array}$ & $\begin{array}{l}\text { Non- } \\
\text { bell } \\
\text { shaped }\end{array}$ & $\begin{array}{c}\text { Non- } \\
\text { bell } \\
\text { shaped }\end{array}$ & Bell shaped & Bell shaped & Bell shaped & Bell shaped & Bell shaped & Bell shaped & Bell shaped \\
\hline $\begin{array}{l}\text { Fractal } \\
\text { characteristics }\end{array}$ & $\begin{array}{l}\text { Single } \\
\text { fractal }\end{array}$ & $\begin{array}{l}\text { Single } \\
\text { fractal }\end{array}$ & $\begin{array}{l}\text { Single } \\
\text { fractal }\end{array}$ & Multifractal & Multifractal & Multifractal & Multifractal & Multifractal & Multifractal & Multifractal \\
\hline
\end{tabular}

(b)

Figure 9: MFDFA and EMD-MFDFA $\alpha-f(\alpha)$ curves of each sub-band of the measured signal and measured noise: (a) MFDFA; (b) EMDMFDFA.

TABle 7: Fractal judgment between signal components $\alpha$ and $f(\alpha)$. 
TABLE 8: Fractal judgment between noise component $\alpha$ and $f(\alpha)$.

\begin{tabular}{|c|c|c|c|c|c|c|c|c|c|c|}
\hline & $\operatorname{nos}-a_{1}$ & nos $-a_{2}$ & $\operatorname{nos}-a_{3}$ & $\operatorname{nos}-a_{4}$ & $\operatorname{nos}-a_{5}$ & $\operatorname{nos}-d_{1}$ & $\operatorname{nos}-d_{2}$ & $\operatorname{nos}-d_{3}$ & $\operatorname{nos}-d_{4}$ & $\operatorname{nos}-d_{5}$ \\
\hline MFDFA & $\begin{array}{c}\text { Non- } \\
\text { bell } \\
\text { shaped }\end{array}$ & $\begin{array}{l}\text { Non- } \\
\text { bell } \\
\text { shaped }\end{array}$ & $\begin{array}{c}\text { Non- } \\
\text { bell } \\
\text { shaped }\end{array}$ & Bell shaped & Bell shaped & Bell shaped & Bell shaped & $\begin{array}{c}\text { Bell } \\
\text { shaped }\end{array}$ & Bell shaped & Bell shaped \\
\hline $\begin{array}{l}\text { EMD- } \\
\text { MFDFA }\end{array}$ & $\begin{array}{l}\text { Non- } \\
\text { bell } \\
\text { shaped }\end{array}$ & $\begin{array}{l}\text { Non- } \\
\text { bell } \\
\text { shaped }\end{array}$ & $\begin{array}{l}\text { Non- } \\
\text { bell } \\
\text { shaped }\end{array}$ & Bell shaped & Bell shaped & Bell shaped & Bell shaped & $\begin{array}{c}\text { Non- } \\
\text { bell } \\
\text {-shaped }\end{array}$ & Bell shaped & Bell shaped \\
\hline $\begin{array}{l}\text { Fractal } \\
\text { characteristics }\end{array}$ & $\begin{array}{l}\text { Single } \\
\text { fractal }\end{array}$ & $\begin{array}{l}\text { Single } \\
\text { fractal }\end{array}$ & $\begin{array}{l}\text { Single } \\
\text { fractal }\end{array}$ & Multifractal & Multifractal & Multifractal & Multifractal & $\begin{array}{l}\text { Single } \\
\text { fractal }\end{array}$ & Multifractal & Multifractal \\
\hline
\end{tabular}

TABLe 9: Difference in Hurst exponent $h(q)$ between the noise component and signal component analyzed by EMD-MFDFA.

\begin{tabular}{|c|c|c|c|c|c|c|c|c|c|c|}
\hline & $a_{1}-$, nos $_{-} \mathrm{a}_{1}$ & $a_{2}-, \operatorname{nos}_{-} a_{2}$ & $a_{3}-\operatorname{nos}_{-} a_{3}$ & $a_{4}-\operatorname{nos}_{-} a_{4}$ & $a_{5}-\operatorname{nos}_{-} a_{5}$ & $d_{1}-\operatorname{nos}_{-} d_{1}$ & $d_{2}-\operatorname{nos} \_d_{2}$ & $d_{3}-\operatorname{nos}_{-} d_{3}$ & $d_{4}-\operatorname{nos}_{-} d_{4}$ & $d_{5}-\operatorname{nos}_{-} d_{5}$ \\
\hline Correlation & -0.970 & -0.593 & 0.958 & 0.959 & 0.971 & 0.977 & 0.995 & 0.989 & 0.994 & 0.981 \\
\hline $\begin{array}{l}\text { Average } \\
\text { error }\end{array}$ & 0.591 & 0.608 & 0.006 & -0.044 & 0.131 & -0.055 & 0.074 & 0.037 & 0.015 & -0.106 \\
\hline $\begin{array}{l}\text { Standard } \\
\text { deviation }\end{array}$ & 0.068 & 0.052 & 0.031 & 0.012 & 0.010 & 0.005 & 0.016 & 0.006 & 0.001 & 0.029 \\
\hline
\end{tabular}

TABLE 10: Difference in $\tau(q)$ between the noise component and signal component analyzed by EMD-MFDFA.

\begin{tabular}{|c|c|c|c|c|c|c|c|c|c|c|}
\hline & $a_{1}-$ nos_a $_{1}$ & $a_{2}-$ nos $\_a_{2}$ & $a_{3}-$ nos $_{-} a_{3}$ & $a_{4}-$ nos $_{-} a_{4}$ & $a_{5}-\operatorname{nos}_{-} a_{5}$ & $d_{1}-$ nos $_{-} d_{1}$ & $d_{2}-$ nos $\_d_{2}$ & $d_{3}-\operatorname{nos}_{-} d_{3}$ & $d_{4}-$ nos_$_{-} d_{4}$ & $d_{5}-$ nos_d $d_{5}$ \\
\hline Correlation & 0.924 & 0.966 & 0.997 & 0.997 & 0.994 & 0.921 & 0.997 & 0.995 & 0.998 & 0.997 \\
\hline Average error & -0.775 & -0.676 & -0.435 & 0.154 & -0.120 & 0.207 & -0.372 & -0.225 & -0.059 & 0.450 \\
\hline $\begin{array}{l}\text { Standard } \\
\text { deviation }\end{array}$ & 3.949 & 4.306 & 0.128 & 0.044 & 0.425 & 0.086 & 0.225 & 0.076 & 0.003 & 0.503 \\
\hline
\end{tabular}

TABLE 11: Difference in $f(\alpha)$ between the noise component and signal component analyzed by EMD-MFDFA.

\begin{tabular}{|c|c|c|c|c|c|c|c|c|c|c|}
\hline & $a_{1}-\operatorname{nos}_{-} a_{1}$ & $a_{2}-\operatorname{nos}_{-} a_{2}$ & $a_{3}-$ nos $_{-} a_{3}$ & $a_{4}-\operatorname{nos}_{-} a_{4}$ & $a_{5}-$ nos $_{-} a_{5}$ & $d_{1}-\operatorname{nos}_{-} d_{1}$ & $d_{2}-\operatorname{nos}_{-} d_{2}$ & $d_{3}-\operatorname{nos}_{-} d_{3}$ & $d_{4}-\operatorname{nos}_{-} d_{4}$ & $d_{5}-\operatorname{nos}_{-} d_{5}$ \\
\hline Correlation & -0.765 & -0.488 & 0.846 & 0.774 & 0.798 & 0.937 & 0.897 & 0.979 & 0.988 & 0.877 \\
\hline Average error & -0.368 & -0.322 & 0.017 & 0.174 & 0.009 & 0.132 & -0.348 & -0.269 & 0.073 & 0.305 \\
\hline $\begin{array}{l}\text { Standard } \\
\text { deviation }\end{array}$ & 0.099 & 0.077 & 0.046 & 0.057 & 0.058 & 0.023 & 0.120 & 0.092 & 0.027 & 0.134 \\
\hline
\end{tabular}

wavelet decomposition of the measured signal and the measured noise. This can be considered as the main noise component, and the results of multifractal analysis show that $a_{4}, a_{5}, d_{4}$, and $d_{5}$ are the main real signal components. The wavelet decomposition components $d_{1}$ and $d_{2}$ of the two channel acquisition signals exhibit disorder. This indicates that the signal is polluted by disordered noise.

The $a_{4}, a_{5}, d_{4}$, and $d_{5}$ components can be used to reconstruct a new signal, regarded as an electrostatic signal after noise filtering. The filtered waveform is shown in Figure 11. It can be seen that the filtered signal is smoother than the original signal, as the interference of power frequency noise and disordered noise has been eliminated, and provides a basis for further signal analysis. Even if the measured noise is completely submerged by noise, the real charge-induced signal can be obtained by filtering, as shown in Figure 12.

The filtered signal was analyzed using EMD-MFDFA, and the $\alpha-f(\alpha)$ curve was found to exhibit multifractal characteristics (see Figure 13). This indicates that the filtered signal has multifractal characteristics. 

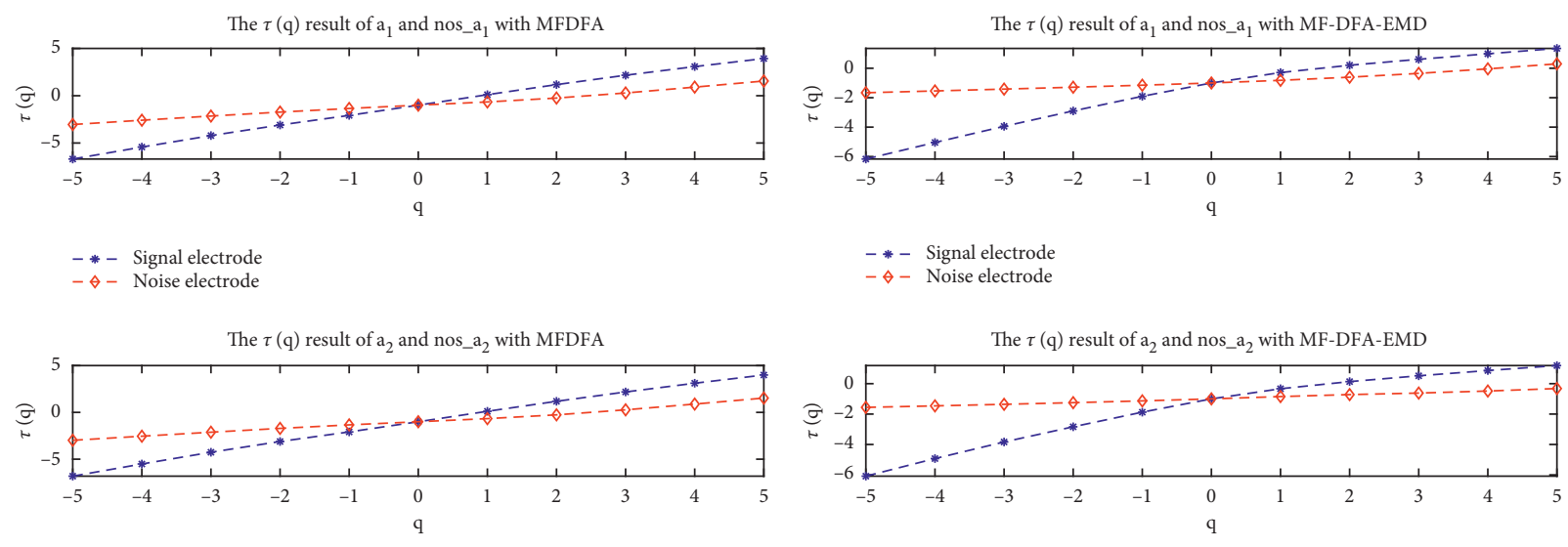

- *- Signal electrode $\begin{array}{ll}-*- & \text { Signal electrode } \\ -\diamond-\text { Noise electrode }\end{array}$

The $\tau$ (q) result of $\mathrm{a}_{3}$ and nos_ $\mathrm{a}_{3}$ with MFDFA

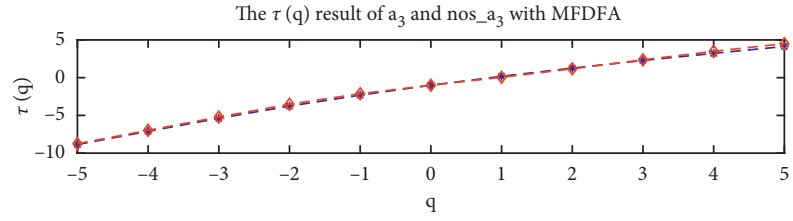

The $\tau$ (q) result of $\mathrm{a}_{3}$ and nos_a $\mathrm{a}_{3}$ with MF-DFA-EMD

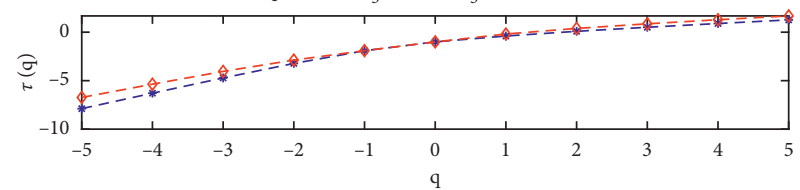

- * - Signal electrode

$-\diamond-$ Noise electrode

\section{-* - Signal electrode}

$\diamond-$ Noise electrode
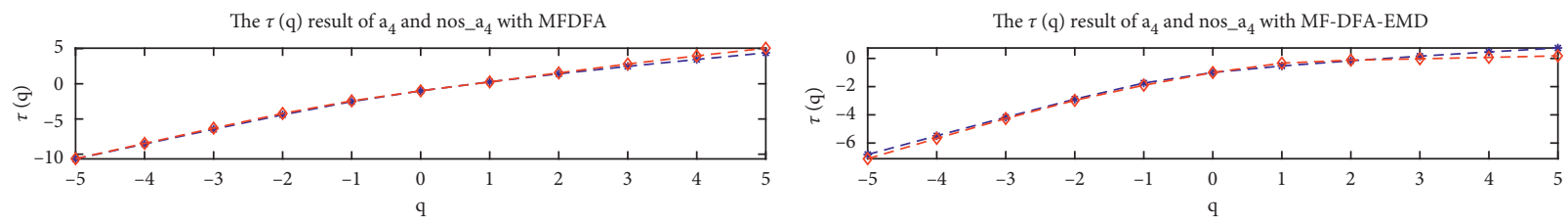

- * - Signal electrode

$-\diamond-$ Noise electrode

- * - Signal electrode

$-\diamond-$ Noise electrode
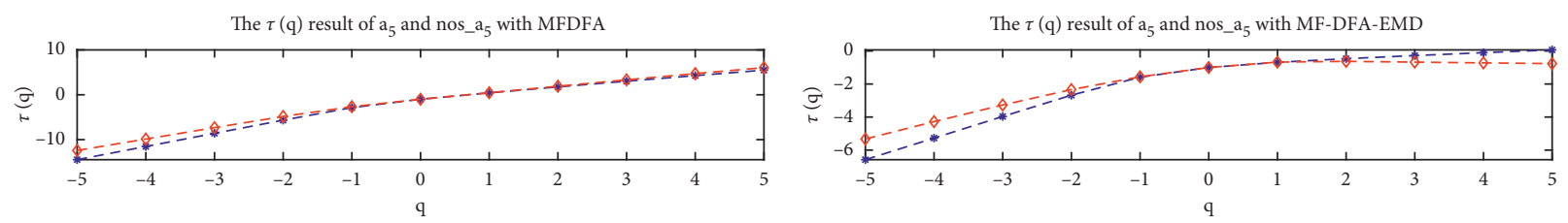

- * - Signal electrode

$-\diamond-$ Noise electrode

- * - Signal electrode

$-\diamond-$ Noise electrode

(a)

Figure 10: Continued. 

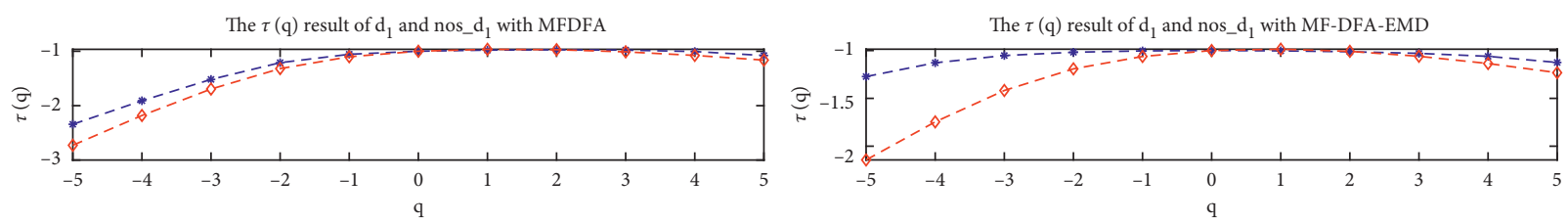

- * - Signal electrode

- * - Signal electrode

$-\diamond-$ Noise electrode
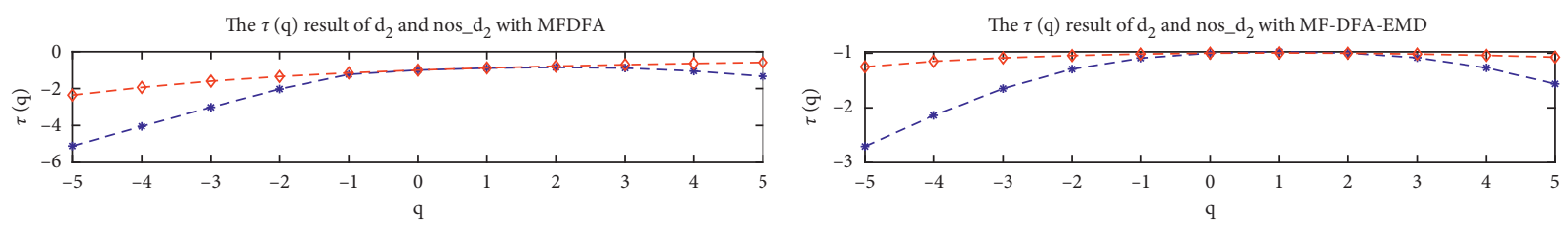

- * Signal electrode

$-\diamond-$ Noise electrode

$-*$ Signal electrode
$-\diamond-$ Noise electrode

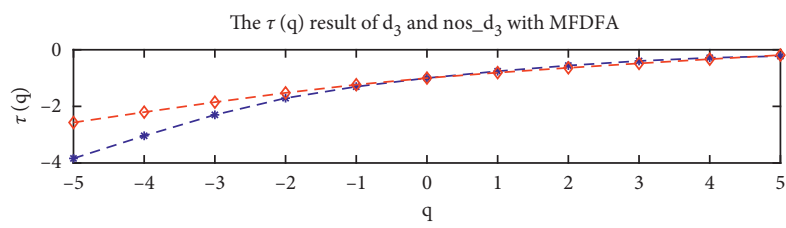

The $\tau$ (q) result of $d_{3}$ and nos_ $d_{3}$ with MF-DFA-EMD

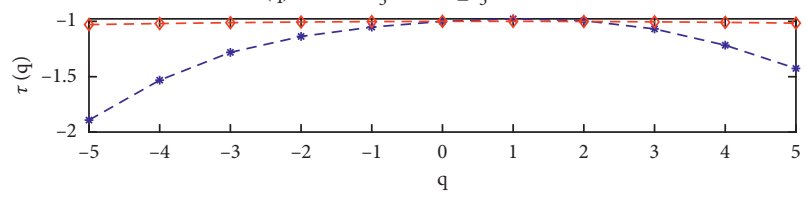

- * - Signal electrode

$\begin{array}{ll}-*- & \text { Signal electrode } \\ -\diamond- & \text { Noise electrode }\end{array}$
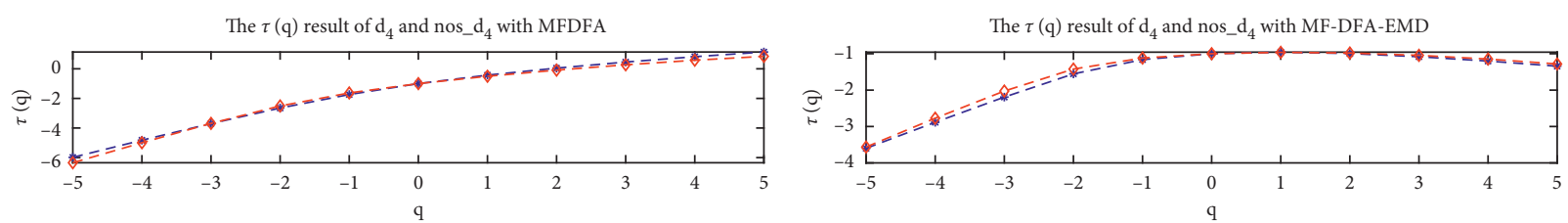

- * - Signal electrode

-* - Signal electrode $-\diamond-$ Noise electrode
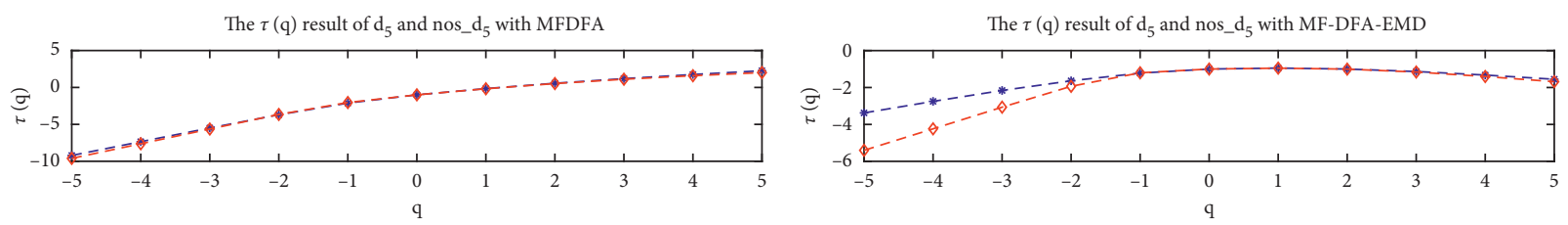

$-*-$ Signal electrode
$-\diamond-$ Noise electrode

$-*-$ Signal electrode
$-\diamond-$ Noise electrode

(b)

FIGURE 10: MFDFA and EMD-MFDFA $q-\tau(q)$ curves of each sub-band of the measured signal and measured noise: (a) MFDFA; (b) EMDMFDFA. 

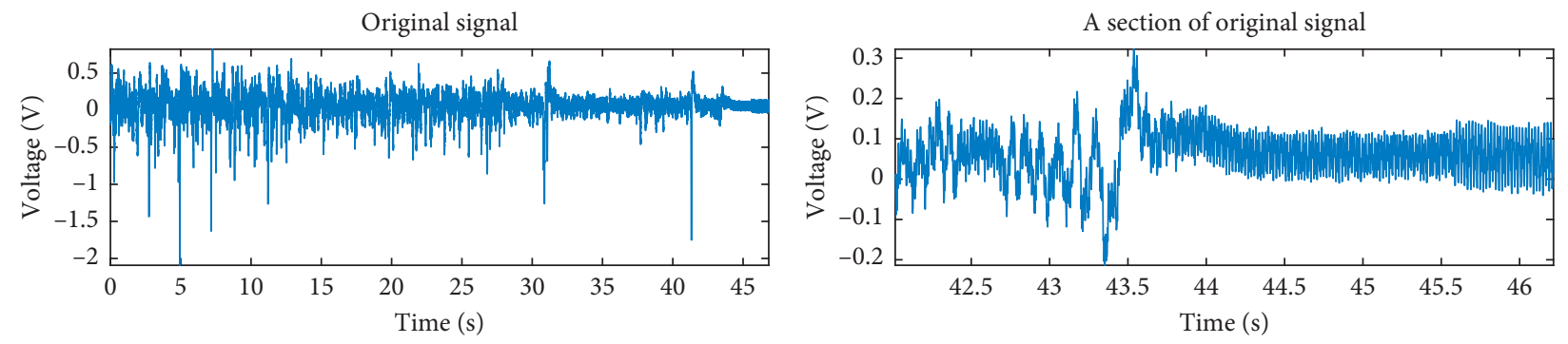

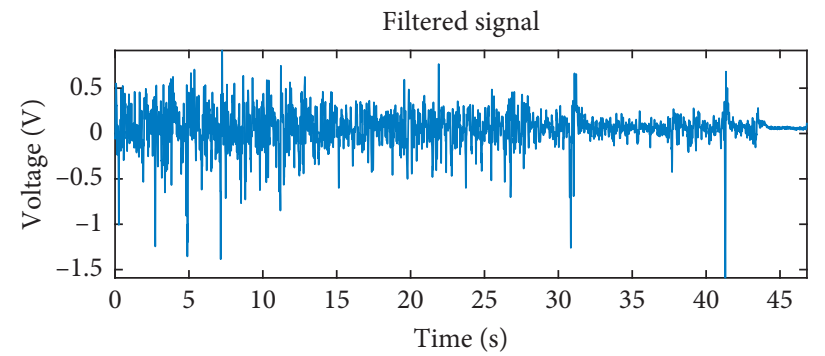

(a)

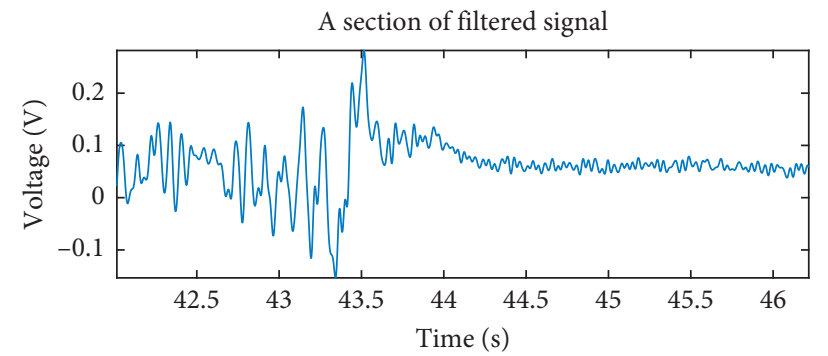

(b)

FIgURE 11: Signal diagram after filtering of the signal-detection electrode.
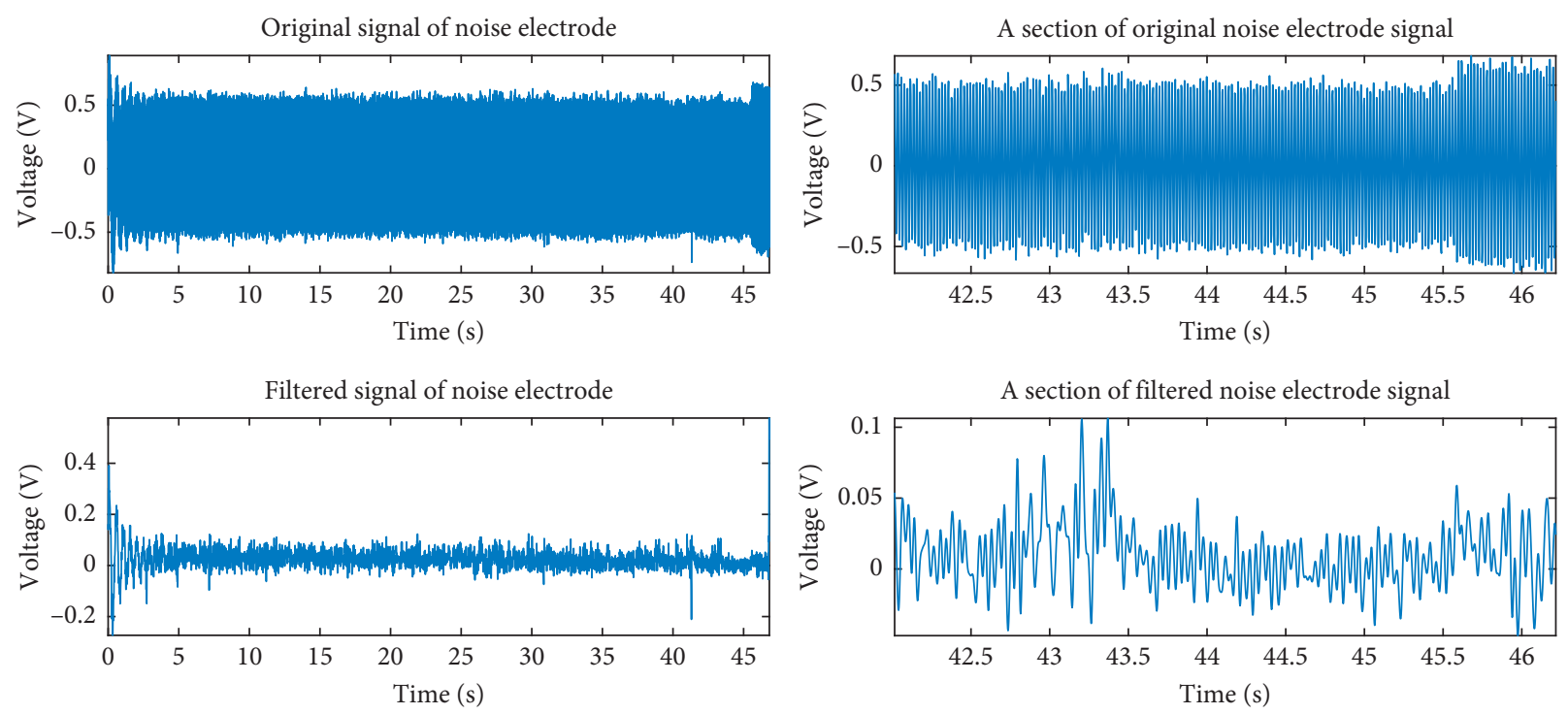

FIGURE 12: Signal diagram after filtering of the noise-detection electrode.

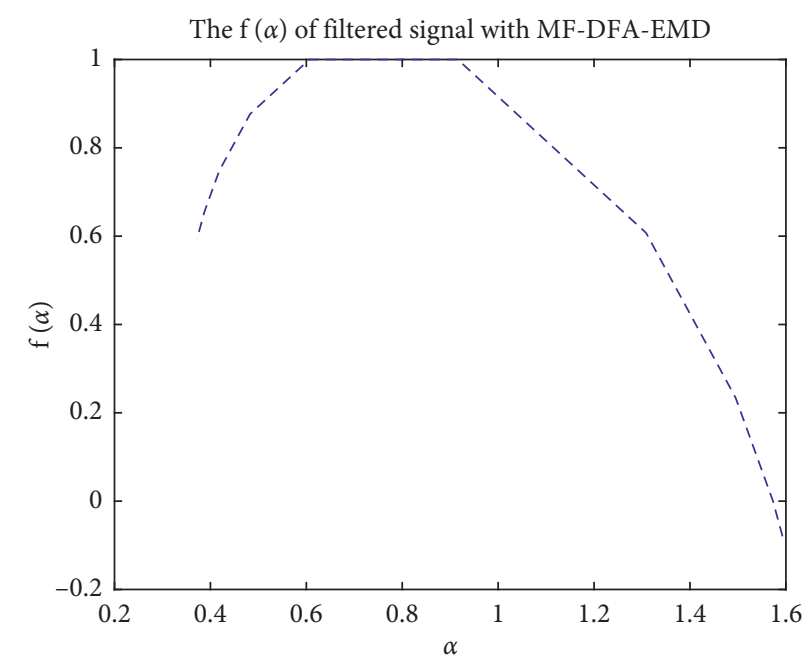

FIGURE 13: $\alpha-f(\alpha)$ curve of the signal after removing the noise. 


\section{Conclusion}

(1) First, the measurement model was improved, and a signal acquisition method using an asymmetric twoelectrode structure was proposed. In this method, large-sized electrodes are used to obtain the induced signals and small-sized electrodes are used to record the noise.

(2) A method of combining EMD-MFDFA with timelapse Pearson correlation analysis has been proposed. Based on the db24 wavelet decomposition method, the measured signal and measured noise were decomposed. The results of the time-lapse Pearson correlation analysis showed that the $d_{3}$ component of the induced signal wavelet decomposition had a strong overall correlation with the noise and exhibited the characteristics of simultaneous changes, without any delay or lag.

(3) Further, based on the wavelet decomposition of the noise signal, time-lapse Pearson correlation analysis found that nos_ $a_{4}-a_{4}, \operatorname{nos}_{-} a_{5}-a_{5}, \operatorname{nos}_{-} d_{3}-d_{3}$, nos $\_d_{1}-d_{1}$, nos $\_a_{3}-a_{3}$, and nos $d_{5}-d_{5}$ exhibited some correlation. However, it was impossible to directly determine which components were noise and which were part of the signal using this method.

(4) Multifractal feature analysis of the measured signal found no obvious multifractal features. MFDFA and EMD-MFDFA analysis of each component after wavelet decomposition did, however, find obvious multifractal features in several components.

(5) Comparing the multifractal results of the measured signal wavelet sub-band and the measured noise wavelet sub-band, there is obvious consistency between nos_ $a_{4}-a_{4}, \quad$ nos_ $a_{5}-a_{5}, \quad$ nos_ $d_{4}-d_{4}$, and nos $d_{5}-d_{5}$. It was verified that the components of the measured signal were mainly concentrated at low frequencies, and $a_{4}, a_{5}, d_{4}$, and $d_{5}$ were considered to be the main components of the real signal.

(6) The signal was reconstructed using components $a_{4}$, $a_{5}, d_{4}$, and $d_{5}$ to obtain the noise-removed induction signal, which presents smoother characteristics. The filtered signal also presents obvious multifractal characteristics.

In future work, the denoised electrostatic induction signal will be used for the measurement of dust concentration. Simultaneously, by combining Ensemble Empirical Mode Decomposition [36] (EEMD), Variational Mode Decomposition [38] (VMD) with stronger ability to extract trends, MFDFA's de-trending capabilities will be further improved.

\section{Data Availability}

The data used to support the findings of this study are included within the supplementary information file.

\section{Conflicts of Interest}

The authors declare that they have no conflicts of interest.

\section{Acknowledgments}

This work was supported by the National Natural Science Foundation of China Program (no. 62073198) and the Major Research Development Program of Shandong Province of China (no. 2016GSF117009).

\section{Supplementary Materials}

"Measured_noise_data.docx" includes the measured noise used in this paper. "Measured_signal_data.docx" includes the measured signal used in this paper. (Supplementary Materials)

\section{References}

[1] J. B. Gajewski, "Electrostatic nonintrusive method for measuring the electric charge, mass flow rate, and velocity of particulates in the two-phase gas-solid pipe flows-its only or as many as 50 Years of historical evolution," IEEE Transactions on Industry Applications, vol. 44, no. 5, pp. 1418-1430, 2008.

[2] J. Li, F. Fu, S. Li, C. Xu, and S. Wang, "Velocity characterization of dense phase pneumatically conveyed solid particles in horizontal pipeline through an integrated electrostatic sensor," International Journal of Multiphase Flow, vol. 76, pp. 198-211, 2015.

[3] X. Qian, D. Shi, Y. Yan, W. Zhang, and G. Li, "Effects of moisture content on electrostatic sensing based mass flow measurement of pneumatically conveyed particles," Powder Technology, vol. 311, pp. 579-588, 2017.

[4] Y. Yan, Y. Hu, L. Wang et al., "Electrostatic sensors-their principles and applications," Measurement, vol. 169, 2021.

[5] X. Qian, Y. Yan, S. Wu, and S. Zhang, "Measurement of velocity and concentration profiles of pneumatically conveyed particles in a square-shaped pipe using electrostatic sensor arrays," Powder Technology, vol. 377, pp. 693-708, 2021.

[6] J. Li, J. Li, X. Cheng, and G. Feng, "Investigation of induced charge mechanism on a rod electrode," Electronics, vol. 8, no. 9, p. 977, 2019.

[7] Y. Hu, Y. Yan, X. Qian, and W. Zhang, “A comparative study of induced and transferred charges for mass flow rate measurement of pneumatically conveyed particles," Powder Technology, vol. 356, pp. 715-725, 2019.

[8] W. Zhang, X. Cheng, Y. Hu, and Y. Yan, "Online prediction of biomass moisture content in a fluidized bed dryer using electrostatic sensor arrays and the Random Forest method," Fuel, vol. 239, pp. 437-445, 2019.

[9] K. Reda and Y. Yan, "Vibration measurement of an unbalanced metallic shaft using electrostatic sensors," IEEE Transactions on Instrumentation and Measurement, vol. 68, no. 5, pp. 1467-1476, 2019.

[10] Y. Yan, L. Wang, T. Wang, X. Wang, Y. Hu, and Q. Duan, "Application of soft computing techniques to multiphase flow measurement: a review," Flow Measurement and Instrumentation, vol. 60, pp. 30-43, 2018.

[11] H. Zhang, "Theoretical prediction of electric fields in windblown sand," Journal of Geophysical Research, vol. 118, 2013.

[12] C. Xu and S. N. Wang, "Electrostatic monitoring of coal velocity and mass flowrate at a power plant," Instrumentation Science \& Technology, vol. 44, 2016.

[13] F. Fu, C. Xu, and S. Wang, "Flow characterization of highpressure dense-phase pneumatic conveying of coal powder 
using multi-scale signal analysis," Particuology, vol. 36, no. 1, 2018.

[14] C. Wang, J. Zhang, W. Zheng, W. Gao, and L. Jia, "Signal decoupling and analysis from inner flush-mounted electrostatic sensor for detecting pneumatic conveying particles," Powder Technology, vol. 305, pp. 197-205, 2017.

[15] K. Reda and Y. Yan, "An improved method for the processing of signals contaminated with strong common-mode periodic noise in correlation velocity measurement," IEEE Sensors Letters, vol. 3, no. 7, pp. 1-4, 2019.

[16] L. Wang, "Radial vibration measurement of rotary shafts through electrostatic sensing and Hilbert-Huang Transform," in Proceedings of the Instrumentation \& Measurement Technology Conference, IEEE, Montevideo, Uruguay, May 2016.

[17] W. Zhang, "Hilbert-huang transform-based electrostatic signal analysis of ring-shape electrodes with different widths," IEEE Transactions on Instrumentation and Measurement, vol. 61, no. 5, pp. 1209-1217, 2012.

[18] C. Wang, N. Zhan, L. Jia, J. Zhang, and Y. Li, "DWT-based adaptive decomposition method of electrostatic signal for dilute phase gas-solid two-phase flow measuring," Powder Technology, vol. 329, pp. 199-206, 2018.

[19] Z. Yan, X. Xu, Y. Wang et al., "Application of ultrasonic Doppler technology based on wavelet threshold denoising algorithm in fetal heart rate and central nervous system malformation detection," World Neurosurgery, vol. 149, pp. 380-387, 2021.

[20] X. Yao, Q. Zhou, C. Wang, J. Hu, and P. Liu, "An adaptive seismic signal denoising method based on variational mode decomposition," Measurement, vol. 177, no. 2, Article ID 109277, 2021.

[21] B. Ji, Z. Hosseini, L. Wang et al., "Spectral wavelet-feature analysis and classification assisted denoising for enhancing magnetic resonance spectroscopy," NMR in Biomedicine, vol. 1, 2021.

[22] S. Wang, J. Na, and Q. Chen, "Adaptive predefined performance sliding mode control of motor driving systems with disturbances," IEEE Transactions on Energy Conversion, vol. 99, p. 1, 2020.

[23] S. Wang, "Asymptotic tracking control for nonaffine systems with disturbances," IEEE Transactions on Circuits and Systems II: Express Briefs, vol. 99, Article ID 3080524, 2021.

[24] J. L. M. Martínez, I. Se Govia-Dominguez, I. Quiros et al., “A modified Multifractal Detrended Fluctuation Analysis (MFDFA) approach for multifractal analysis of precipitation [J]," Physica A: Statistical Mechanics and Its Applications, vol. $565,2020$.

[25] A. K. Maity, R. Pratihar, A. Mitra et al., "Multifractal Detrended Fluctuation Analysis of alpha and theta EEG rhythms with musical stimuli," Chaos, Solitons \& Fractals, vol. 81, no. 4, pp. 52-67, 2015.

[26] O. E. Dick and I. A. Svyatogor, "Wavelet and multifractal estimation of the intermittent photic stimulation response in the electroencephalogram of patients with dyscirculatory encephalopathy," Neurocomputing, vol. 165, pp. 361-374, 2015.

[27] J. W. Kantelhardt, S. A. Zschiegner, E. Koscielny-Bunde, S. Havlin, A. Bunde, and H. E. Stanley, "Multifractal detrended fluctuation analysis of nonstationary time series," Physica A: Statistical Mechanics and Its Applications, vol. 316, no. 1-4, pp. 87-114, 2002.

[28] L. Minhyuk, J. W. Song, K. Sondo et al., "Asymmetric market efficiency using the index-based asymmetric-MFDFA,"
Physica A: Statistical Mechanics and Its Applications, vol. 512, pp. 1278-1294, 2018.

[29] A. Ji, B. Sha, B. Au et al., "Effect of missing data on short time series and their application in the characterization of surface temperature by detrended fluctuation analysis," Computers \& Geosciences, vol. 153, 2021.

[30] J. Lin and C. Dou, "Y Liu, Multifractal detrended fluctuation analysis based on optimized empirical mode decomposition for complex signal analysis," Nonlinear Dynamics, vol. 9, 2021.

[31] M. Huang and Z. Liu, "Research on mechanical fault prediction method based on multifeature fusion of vibration sensing data," Sensors, vol. 20, no. 1, 2019.

[32] Z. Nan, G. Hu, X. Zhang et al., "Wrist pulse recognition based on multi-fractal spectrum," in Proceedings of the International Congress on Image \& Signal Processing, IEEE, Las Vegas, NV, USA, August 2016.

[33] G. Gaurav, R. S. Anand, and V. Kumar, "EEG based cognitive task classification using multifractal detrended fluctuation analysis," Cognitive Neurodynamics, vol. 111, pp. 1-15.

[34] J. Li, X. Ma, M. Zhao et al., "A novel MFDFA algorithm and its application to analysis of harmonic multifractal features," Electronics, vol. 8, no. 2, 2019.

[35] N. E. Huang, Z. Shen, S. R. Long et al., "The empirical mode decomposition and the Hilbert spectrum for nonlinear and non-stationary time series analysis," Proceedings of the Royal Society of London. Series A: Mathematical, Physical And Engineering Sciences, vol. 454, pp. 903-995, 1998.

[36] G. Rilling, P. Flandrin, and P. Gonçalves, "On empirical mode decomposition and its algorithms," in Proceedings of the IEEEEURASIP Workshop on Nonlinear Signal and Image Processing, pp. 8-11, Grado, Italy, June 2003.

[37] J. F. Muzy, "Random cascade model in the limit of infinite integral scale as the exponential of a non-stationary $\$ 1 / \mathrm{f} \$$ noise," Application to Volatility Fluctuations in Stock Markets, vol. 87, no. 4-1, 2013.

[38] Z. Wu and N. E. Huang, "Ensemble empirical mode decomposition: a noise-assisted data analysis method," Advances in Adaptive Data Analysis, vol. 1, no. 01, 2011. 